\title{
On Using Anisotropic Diffusion for Skeleton Extraction
}

\author{
Cem Direkoglu • Rozenn Dahyot • Michael Manzke
}

Received: 4 October 2010 / Accepted: 19 May 2012 / Published online: 13 June 2012

(C) Springer Science+Business Media, LLC 2012

\begin{abstract}
We present a novel and effective skeletonization algorithm for binary and gray-scale images, based on the anisotropic heat diffusion analogy. We diffuse the image in the direction normal to the feature boundaries and also allow tangential diffusion (curvature decreasing diffusion) to contribute slightly. The proposed anisotropic diffusion provides a high quality medial function in the image: it removes noise and preserves prominent curvatures of the shape along the level-sets (skeleton features). The skeleton strength map, which provides the likelihood of a point to be part of the skeleton, is defined by the mean curvature measure. Finally, thin and binary skeleton is obtained by non-maxima suppression and hysteresis thresholding of the skeleton strength map. Our method outperforms the most related and the popular methods in skeleton extraction especially in noisy conditions. Results show that the proposed approach is better at handling noise in images and preserving the skeleton features at the centerline of the shape.
\end{abstract}

Keywords Skeletonization - Feature extraction · Heat flow $\cdot$ Computer vision

\section{Introduction}

Skeleton or medial axis (Blum 1967) is a thin version of the shape, which is an important feature for shape description in

C. Direkoglu ( $\varangle) \cdot$ R. Dahyot · M. Manzke

School of Computer Science and Statistics, Trinity College

Dublin, Dublin, Ireland

e-mail: cdirekoglu@googlemail.com

R. Dahyot

e-mail: rozenn.dahyot@scss.tcd.ie

M. Manzke

e-mail: michael.manzke@cs.tcd.ie image processing and computer vision. It offers simple and compact representation of shapes while preserving its topology. Skeletons can be used for instance to classify objects (Aslan et al. 2008; Ward and Hamarneh 2010) or to estimate their poses and orientations (Macrini et al. 2008). There are different algorithms for skeletonization of shapes in images. We review skeletonization techniques divided into two categories: the ones that are limited to the pre-segmented binary images (Sect. 1.1) and the ones that are capable of extracting skeletons from gray-scale images (Sect. 1.2).

\subsection{Skeletonization of Binary Images}

Popular techniques used for skeleton extraction in binary images are based on thinning (Lam et al. 1992), Voronoi diagrams (Ogniewicz and Kubler 1995), distance transform (Arcelli and Baja 1992; Kimmel et al. 1995; Malandain and Vidal 1998), Poisson equation (Gorelick et al. 2006), Newton's law (Siddiqi et al. 1999) and Electrostatic field (Grogorishin et al. 1996). Thinning (Lam et al. 1992) is a process that deletes object boundary pixels iteratively with a set of conditions. Complex conditions are required to terminate this deleting process as well as to preserve the topology and the connectivity of the skeleton. In Ogniewicz and Kubler (1995), the skeleton is extracted from the Voronoi diagram computed on the boundary of the object. This approach is theoretically well defined in a continuous space, which means a high sampling rate of the boundary points is required to compute a Voronoi diagram of good quality. The Voronoi skeletons also need complex post-processing stages to prune the branches. Recently, Krinidis and Chatzis (2009) proposed a physics-based deformable model for skeletonization that does not produce spurious branches.

A medial function is a scalar function that locally assigns higher values to skeleton points than non-skeleton points. 
Many techniques use distance transform as a medial function to extract skeletons (Arcelli and Baja 1992; Kimmel et al. 1995; Malandain and Vidal 1998). The distance transform computes at each pixel position its minimum distance to the shape boundary. However, the distance transform is very sensitive to the small perturbations of the boundary, since each value on shape is assigned according to the single boundary point (nearest one). Similar to the Voronoi skeletons, the skeletons obtained by the distance transform need a pruning stage if the boundary is noisy (Bai et al. 2007; Shen et al. 2011). To overcome the limitations of the distance transform, a few smooth medial functions have been introduced which are based on Newton's law (Siddiqi et al. 1999), Electrostatic field (Grogorishin et al. 1996) and Poisson equation (Gorelick et al. 2006). These methods are taking into account several boundary points and therefore they reflect better the global properties of the shape than the distance transform does. However all these methods presented above require pre-segmented binary images, since they use object boundary to compute skeletons.

\subsection{Skeletonization of Gray-Level Images}

Tari et al. (1997) extract skeletons from gray-scale images by analysing the level-set curves of the edge strength function. The edge strength function is computed using the linear diffusion equation. Lindeberg (1998) treated the skeletonization in a similar way as edge detection in scale-space (Witkin 1983), with automatic scale selection. Scale-space analysis involves generating images at coarser resolution by convolving the original image with a Gaussian kernel. Koenderink (1984) and Hummel (1986) pointed out that the family of derived images using the Gaussian kernel may be equivalently viewed as the solution of the linear heat diffusion equation. In linear heat diffusion, blurring is required to be spatially invariant which makes it difficult to obtain accurate locations of edges and skeleton features at coarse scales. Linear diffusion techniques can cause loss and relocation of skeleton features, which is an important drawback.

There are also approaches which uses gradient vector diffusion for skeleton extraction in gray-scale images. The first gradient vector diffusion was introduced by $\mathrm{Xu}$ and Prince (1998) as a new external force model for active contours in the shape extraction context. Then, this model has been adapted for skeleton extraction (Yu and Bajaj 2004; Le Bourgeois and Emptoz 2007). Yu and Bajaj (2004) compute skeletons in gray-level images based on anisotropic gradient vector diffusion, where the initial vector fields are obtained by various ways to overcome noise in images. However, gradient-based approaches, which are also used in active contour models, are sensitive to noise (Direkoglu and Nixon 2007). Image smoothing or some heuristics (Yu and Bajaj 2004) can be applied to improve initial gradient vector field. However, they can only increase the tolerance to the noise and they are likely to lose important features after smoothing.

\subsection{Contribution}

Given a smooth medial function, the curvature maxima along the level-sets define the shape skeleton (Tari et al. 1997; Lindeberg 1998). In this paper, we propose a novel medial function, which can be computed both for binary and gray-scale images, based on the anisotropic heat diffusion. We diffuse the image in the normal direction to the feature boundaries and also allow the tangential diffusion (curvature decreasing diffusion) to make small contribution. A diffusion coefficient is used to control the relative weight of the tangential diffusion with respect to the normal diffusion, defining a family of medial functions controlled by a unique parameter. Our approach denoises the image and object boundary, while preserving the prominent curvatures of the shape along the level-sets that represent skeleton locations. Once the medial function is computed with the proposed anisotropic diffusion, the skeleton strength map is then defined by the mean curvature measure. The overall process is terminated by removing non-maxima and by using hysteresis thresholding to obtain a thin and binary skeleton.

The proposed method performs better than the most related diffusion methods and better than the other popular methods in skeleton extraction especially in noisy conditions. The proposed diffusion removes noise in images and on the object boundaries, while preserving the skeleton features at the centerline of the shape. This has been validated both with quantitative and qualitative (visual) evaluations. We directly diffuse images without the need of any segmentation or shape extraction. We do not need to compute edge maps or initialize boundaries of the shape like most of the other methods in skeleton extraction. In addition, we do not need any skeleton pruning method as a post-processing stage, since the proposed diffusion does not produce any spurious branches.

An earlier version of this work was presented in Direkoglu et al. (2010). This paper extends further the evaluations and the comparisons. In particular, we assess the accuracy and robustness to different noise types and noise levels in the images, while comparing with other related methods. We also study the effect of an increasing diffusion time and the diffusion coefficient.

The rest of the paper is organized as follows. Section 2 introduces the proposed anisotropic heat diffusion and the novel medial function. Section 3 presents our experimental results and the comparisons with other techniques. Concluding remarks are in Sect. 4. 
Fig. 1 Directions to the feature boundary through a given point

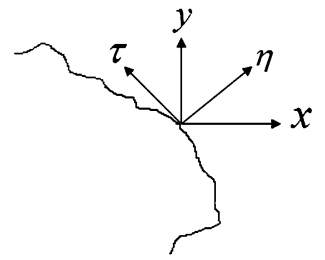

\section{Proposed Diffusion and Skeleton Extraction}

In skeleton extraction, it is an important task to compute a smooth medial function of high quality that can provide skeleton features well and without noise. One of the main features for skeleton point detection is curvature maxima along the level-sets of the medial function. In this section, we present the proposed anisotropic heat diffusion as a novel medial function generation technique, which can remove noise in the image and can preserve the prominent curvatures of the object shape along the level-sets. Introductory tutorials on the heat diffusion equation are also given in Ursell (2007) and Direkoglu (2009). The proposed anisotropic heat diffusion equation is obtained with the following considerations.

Edge directions are related to the tangents of the feature boundaries of an image $I$. Let $\eta$ denote the direction normal to the feature boundary through a given point (the gradient direction), and let $\tau$ denote the tangent direction, as shown in Fig. 1. These directions can be written in terms of the first derivatives of the image, $I_{x}$ and $I_{y}$, as

$\eta=\frac{\left(I_{x}, I_{y}\right)}{\sqrt{I_{x}^{2}+I_{y}^{2}}}, \quad \tau=\frac{\left(-I_{y}, I_{x}\right)}{\sqrt{I_{x}^{2}+I_{y}^{2}}}$

Since $\eta$ and $\tau$ constitute orthogonal directions, the rotationally invariant Laplacian operator can be expressed as the sum of the second order spatial derivatives, $I_{\eta \eta}$ and $I_{\tau \tau}$, in these directions and the linear heat conduction equation can be written as follows,

$\frac{\partial I}{\partial t}=\nabla^{2} I=\left(I_{\eta \eta}+I_{\tau \tau}\right)$

In the heat equation, $\partial I / \partial t$ represents the rate of change of image intensity values (with respect to time) and $\nabla^{2}$ is spatial Laplacian operator. Omitting the normal diffusion, while keeping the tangential diffusion yields the well known Geometric Heat Flow (GHF) equation (Kimia and Siddiqi 1994) as

$\frac{\partial I}{\partial t}=I_{\tau \tau}=\frac{\left(I_{x x} I_{y}^{2}-2 I_{x y} I_{x} I_{y}+I_{y y} I_{x}^{2}\right)}{\left(I_{x}^{2}+I_{y}^{2}\right)}$

GHF is an anisotropic diffusion and is widely used for image denoising and enhancement. It diffuses along the boundaries of image features, but not across them. It removes noise in the image and preserves prominent edges. It derives its name from the fact that, under this flow, the feature boundaries of the image evolve in the normal direction in proportion to their mean curvature $\kappa$ as given below

$$
\begin{aligned}
\frac{\partial I}{\partial t} & =I_{\tau \tau}=\frac{\left(I_{x x} I_{y}^{2}-2 I_{x y} I_{x} I_{y}+I_{y y} I_{x}^{2}\right)}{\left(I_{x}^{2}+I_{y}^{2}\right)^{3 / 2}}\left(I_{x}^{2}+I_{y}^{2}\right)^{1 / 2} \\
& =\kappa \mid \nabla I
\end{aligned}
$$

Thus GHF decreases the curvature of level-sets of the image while removing noise to obtain sharp edges.

On the other hand, omitting the tangential diffusion, while keeping the normal diffusion in the heat equation yields

$\frac{\partial I}{\partial t}=I_{\eta \eta}=\frac{\left(I_{x x} I_{x}^{2}+2 I_{x y} I_{x} I_{y}+I_{y y} I_{y}^{2}\right)}{\left(I_{x}^{2}+I_{y}^{2}\right)}$

Normal diffusion is also an anisotropic diffusion. It diffuses across the edges in the image and does not preserve them. Because of this property, it did not take much attention for image smoothing and enhancement or for edge detection. Significant application of the normal diffusion can only be observed in Manay and Yezzi (2003) for image segmentation. In this application, the normal diffusion is called Anti-Geometric Heat Flow (A-GHF), since the diffusion is in the orthogonal direction to the GHF.

Here, we choose the normal diffusion as a main tool for medial function generation in binary and gray-scale images for skeleton extraction purpose. The normal diffusion can preserve prominent curvatures of the shapes along the levelsets in images while removing noise, since it does not diffuse along the level-sets (in tangent direction). However, we do not completely omit the tangential diffusion and let it contribute slightly depending on the user, since it can also remove noise along the feature boundaries and contribute to obtaining a smoother skeleton. The proposed anisotropic heat diffusion problem for medial function generation is given below,

$$
\frac{\partial I}{\partial t}=I_{\eta \eta}+c I_{\tau \tau}
$$

with $\mid \begin{aligned} & I(\mathbf{x}, t=0)=F(\mathbf{x}), \quad \text { initial condition } \\ & \frac{\partial I(\mathbf{x}, t)}{\partial n}=0, \quad \text { boundary condition }\end{aligned}$

where $c$ is the diffusion coefficient which is a positive constant and which must be small to prevent excess smoothing in the direction tangent. In our experiments $c=0.2,0.1$ or $0 . \mathbf{x}=(x, y)$ is the space vector and $F(\mathbf{x})$ is the input binary or gray-scale image, which represents the initial condition of the diffusion problem. The boundaries of the image are insulated with homogeneous Neuman condition, $d I / d n=0$, which means there is no heat flow in, or out of the image domain definition. In boundary condition, $n$ represents the direction normal to the image boundary.

The skeleton strength map (SSM) is the likelihood for each pixel to be a skeleton point. In our algorithm, the SSM 
is the mean curvature measure of the level-sets that is computed after terminating diffusion,

$S S M=\kappa\left(\mathbf{x}, t_{s}\right)=\nabla \cdot\left(\frac{\nabla I\left(\mathbf{x}, t_{s}\right)}{\left|\nabla I\left(\mathbf{x}, t_{s}\right)\right|}\right)$

where the $S S M<0$ represents $S S M$ for brighter regions and the $S S M>0$ represents $S S M$ for darker regions. $t_{s}$ is the number of iterations (diffusion time). For binary images without any noise, the proposed diffusion can be terminated automatically when the maximum intensity value becomes less than a threshold (such as less than 0.99). For noisy images the selection of $t_{s}$ depends on user and it is determined from the noise level in the image. The overall process is completed by non-maxima suppression to make SSM thin, and hysteresis thresholding to observe binary skeleton.

\section{Experimental Results and Discussions}

In this section, we first present the qualitative evaluations, then the quantitative evaluations and finally illustrate some examples on gray-scale images.

\subsection{Qualitative (Visual) Evaluations}

We present the comparison of the proposed diffusion with distance transform, Poisson equation and Gaussian filtering based techniques for medial function generation. The comparison is done on a flower shape image of size $228 \times 226$, which is a pre-segmented binary image with perturbations on the boundary as shown in Fig. 2.

Figures 3(a)-(f) show the medial function generation on the flower shape object by the distance transform (a), Poisson equation (b), Gaussian filtering (c), the proposed diffusion with $c=0.2(\mathrm{~d})$, the proposed diffusion with $c=0.1$ (e) and the proposed diffusion with $c=0$ (f). The first column in Figs. 3(a)-(f) show the medial function on the flower shape object by the algorithms. The second column in Figs. 3(a)-(f) illustrate the level-sets of the medial functions. The third column in Figs. 3(a)-(f) show the computed $S S M<0$, which represents the negative values of the mean curvature measure of the level-sets. In this evaluation, the $S S M<0$ shows the quality of a medial function for skeleton extraction. The last column in Figs. 3(a)-(f) illustrates the $S S M<0$ and the object in the same image.

Fig. 2 Flower shape image of size $228 \times 226$ with perturbations on the boundary

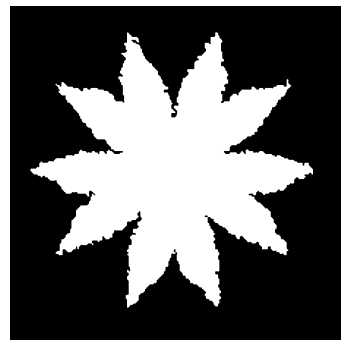

The distance transform (Arcelli and Baja 1992; Kimmel et al. 1995; Malandain and Vidal 1998) computes at each shape point its nearest distance to the shape boundary. Since the distance transform assigns each value on shape according to the single boundary point, it is observed that the levelsets obtained by the distance transform, especially near the boundary, are not smooth and sensitive to the small perturbations of the boundary as shown in Fig. 3(a). It is also seen that the $S S M<0$ is affected by noisy boundary and it creates branches on the skeleton. The distance transform is limited to pre-segmented binary images, since it depends on shape boundary.

The Poisson equation arises in gravitation and electrostatics. It is a linear diffusion with a steady state and a unique solution that has been introduced for shape representation and skeleton extraction (Gorelick et al. 2006). In Fig. 3(b), it is observed that level-sets in the interior regions of the object are very smooth and the curvatures that define the skeleton locations are almost lost. The level-sets close to the boundary of the object are also affected by the noise on the boundary. The $S S M<0$ is quite blurry in the regions inside the flower shape and the skeleton features are lost. There is also noise close to the object boundary. Similarly to the distance transform, the Poisson equation requires the extraction of the shape boundary and therefore can only be applied to binary images.

Gaussian scale-space (Lindeberg 1998) is also used for skeletonization that can be applied to both binary and grayscale images. Gaussian filtering can be equivalently viewed as the solution of linear heat diffusion equation, which is a direction and space invariant diffusion. Figure 3(c) shows the medial function generation by the linear diffusion after diffusion time $t_{s}=250$. From the level-sets, it is observed that linear heat diffusion removes noise on the boundary. However, it also smoothes prominent curvatures of the shape and causes blurry skeleton features, which are almost lost $(S S M<0)$. If we increase the diffusion time, we will lose skeleton features more. On the other hand if we decrease diffusion time, the diffusion may not be enough to generate a medial function and to remove noise on the boundary.

Figure 3(d) shows the proposed diffusion for skeleton extraction with $c=0.2$ and $t_{s}=250$, which is equal to the diffusion time in the linear heat flow in Fig. 3(c). It is observed that the proposed diffusion removes noise on the boundary and preserves prominent curvatures of the shape along the level-sets, which represent skeleton features. The $S S M<0$ highlights skeleton points very well in comparison to the Poisson equation and Gaussian filtering. In addition, there is no noise close to the object boundary in comparison to the distance transform and Poisson equation. Figure 3(e) and (f) also show the proposed diffusion with parameter values $c=0.1$ and $c=0$, respectively. The diffusion time $t_{s}=250$, which is same as the diffusion time in the linear heat flow 
Fig. 3 Comparison of the proposed diffusion $(\mathbf{d}, \mathbf{e}, \mathbf{f})$ with the distance transform (a), Poisson equation (b) and Gaussian filtering (c) with their medial functions (1st column), their level sets (2nd column), their negative values of the mean curvature of the level sets $(S S M<0)$, and $S S M<0$ superimposed with the original shape
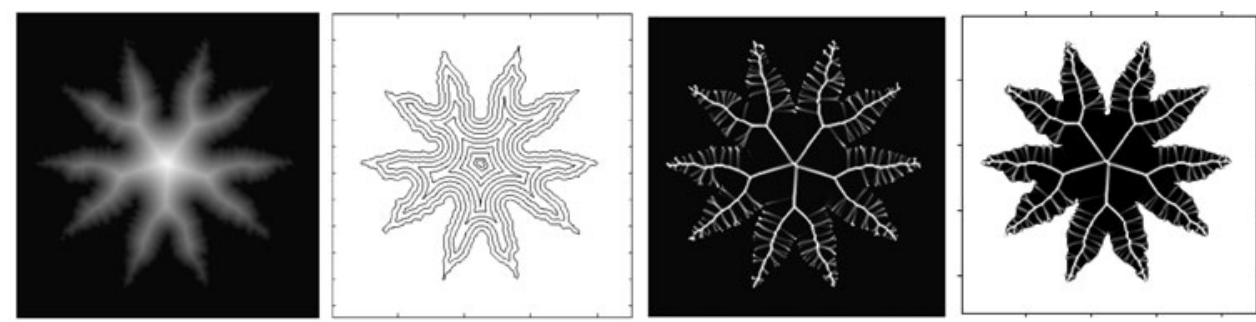

(a) Distance transform (Arcelli and Baja (1992); Kimmel et al. (1995); Malandain and Vidal (1998))
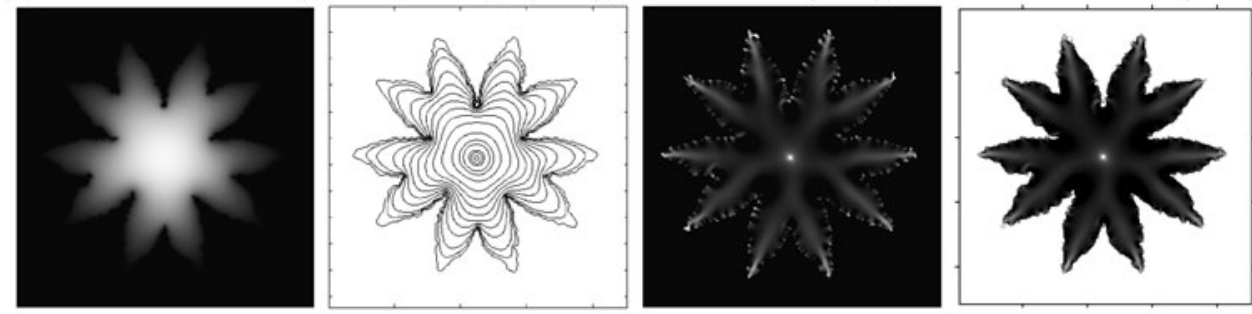

(b) Poisson equation (Linear diffusion) (Gorelick et al. (2006))
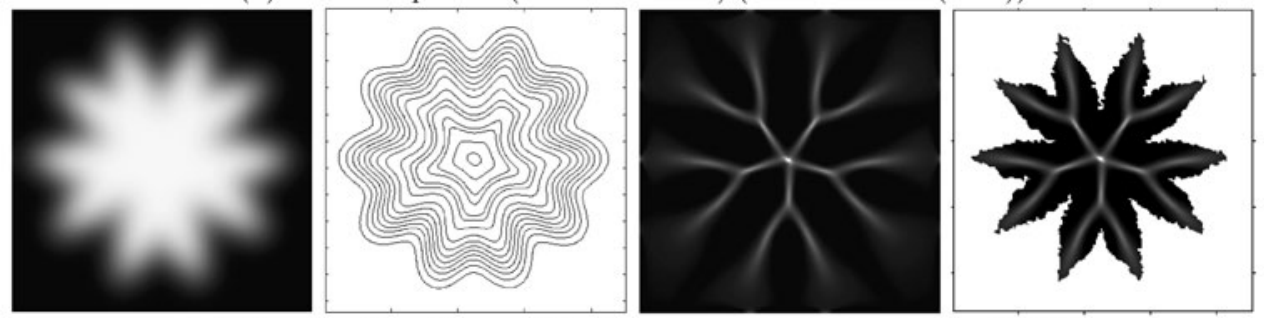

(c) Gaussian filter (Linear diffusion with $t_{s}=250$ ) (Lindeberg (1998))
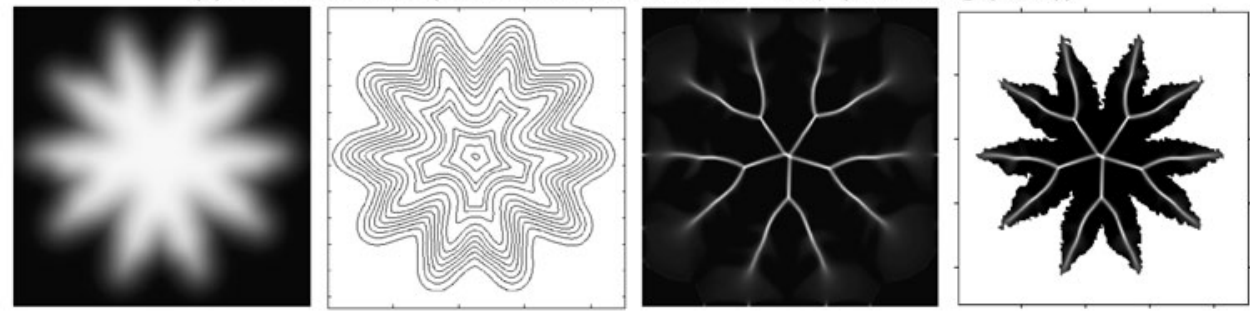

(d) Proposed diffusion with $c=0.2$ and $t_{s}=250$
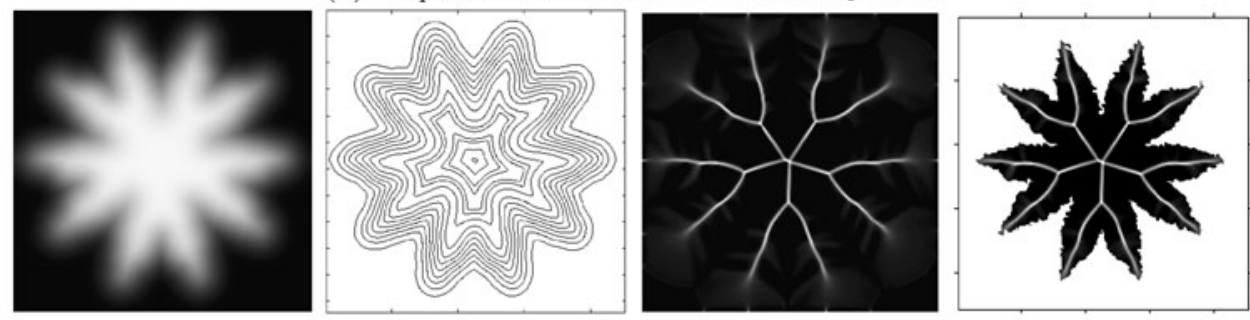

(e) Proposed diffusion with $c=0.1$ and $t_{s}=250$
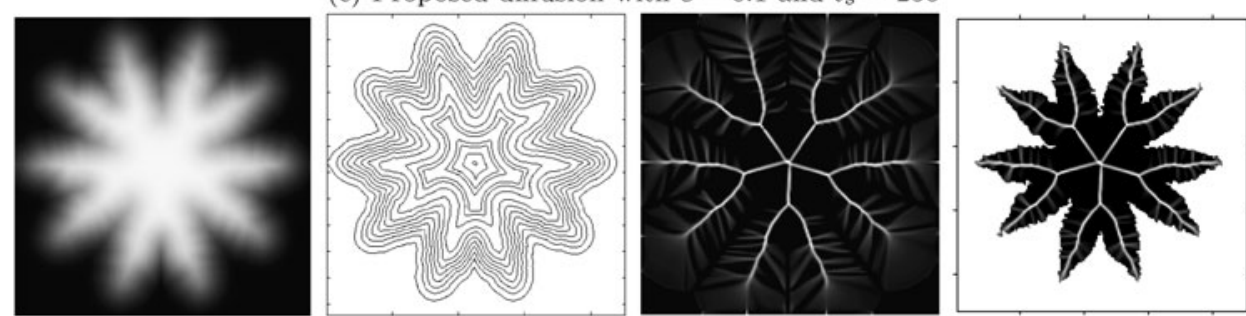

(f) Proposed diffusion with $c=0$ and $t_{s}=250$ in Fig. 3(c) and same as the diffusion time in the proposed diffusion with parameter value $c=0.2$ in Fig. 3(d). From the level-sets, it is observed that as the value of diffusion coefficient decreases, prominent curvatures become sharper, while removing noise. Choosing parameter values $c=0.2$ and $c=0.1$ provides a smoother skeleton than $c=0$, since 
Fig. 4 Obtaining thin and binary skeleton for $c=0.2$.

(a) Non-maxima suppression skeleton strength map $(S S M<0)$, (b) binary skeleton after hysteresis thresholding, (c) skeleton with shape boundary

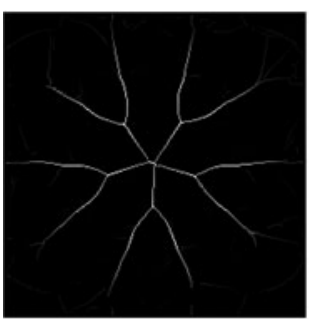

(a)

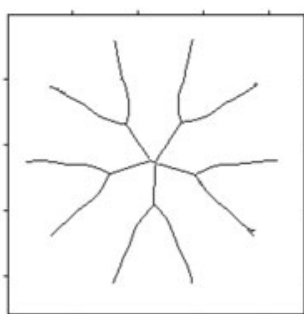

(b)

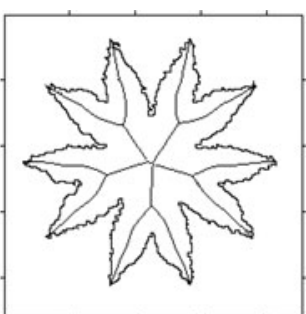

(c) a small amount of tangential diffusion smoothes along the level sets.

Once we obtain the $S S M<0$ with $c=0.2$, which is shown in the third column of Fig. 3(d), the overall process is completed by non-maxima suppression to make the skeleton thin and hysteresis thresholding to obtain a binary skeleton as illustrated in Fig. 4(a) and (b), respectively. Figure 4(c) also shows the skeleton and shape of the object in the same image.

\subsubsection{Impact of Diffusion Time $t_{s}$}

We investigate the impact of the diffusion time (number of iterations) on the proposed diffusion, on the linear diffusion method (Lindeberg 1998), as well as on the Anisotropic Gradient Vector Diffusion (AGVD) method (Yu and Bajaj 2004) for comparison purpose. In AGVD method, the initial gradient vector field is obtained using some heuristics to overcome noise in the image, as described in their original paper. Then the anisotropic diffusion is applied to the initial vector field, where the diffusion coefficients, in each direction, are determined based on the angle information between the central vector and the surrounding vectors. A skeleton strength map is computed from the diffused vector field, and finally non-maxima suppression and hysteresis thresholding is applied to obtain binary skeleton. There are two parameters, $K$ and $t_{s}$, in the AGVD, where the $K$ controls the amount of diffusion in each direction together with the angle information, and the $t_{s}$ is the diffusion time (iterations). In addition, the definition of the skeleton strength map (SSM) in the AGVD is different from the definition of the $S S M$ in the proposed image diffusion. In our algorithm, the $S S M$ is the mean curvature measure after the proposed image diffusion, and the $S S M$ for the brighter regions is denoted by the $S S M<0$. In AGVD, the $S S M$ for the brighter regions is computed using the diffused vector field with a heuristic formulation. The $S S M$ obtained by the AGVD model will be denoted by SSAG in the evaluations.

The comparison is done on a binary image of human shape of size $350 \times 335$ that is shown in the first column of Fig. 5(a). Figures 5(a)-(d) show the $S S M<0$ for the linear diffusion (a) the SSAG for the AGVD method with $K=4$ (b), the $S S M<0$ for the proposed diffusion with $c=0.2$ (c) and the $S S M<0$ for the proposed diffusion with $c=0$ (d). In Figs. 5(a)-(d), the images from left to right show the skeleton strength maps for brighter regions with respect to increasing diffusion times $t_{s}=300,500,700$ and 900. In linear diffusion, it is observed that as $t_{s}$ increases, the skeleton features become blurry and some parts such as the head, shoulders, hands, upper legs and feet are almost lost. In AGVD, some parts such as the head become blurry, as well as the skeleton grows outside the shape from the hand and from the feet. In the proposed diffusion with $c=0.2$, it is observed that as the number of iterations increases, skeleton features are preserved. It is also seen that the proposed diffusion with parameter value $c=0$ preserves the skeleton features better than the proposed diffusion with parameter value $c=0.2$. However, choosing $c=0$ is more sensitive to the small variations on the boundary than choosing $c=0.2$. It is better to keep a small amount of tangential diffusion ( $c=0.2$ or $c=0.1$ ), since it helps to handle small perturbations and noise on the boundaries. Once the $S S M<0$ is computed after the proposed diffusion with parameter values $c=0.2$ and $t_{s}=300$, thinning (non-maxima suppression) and hysteresis thresholding is applied to obtain a binary skeleton as shown in Fig. 6(a). Figure 6(b) also shows the skeleton with the superimposed object shape.

\subsubsection{Noisy Images}

Our algorithm is also assessed on noisy images. The same experiments are conducted using the linear diffusion (Lindeberg 1998) and using the AGVD (Yu and Bajaj 2004) for comparison purpose. For all of the diffusion models, we compute the skeleton strength map for the brighter regions and then apply non-maxima suppression and hysteresis thresholding to obtain thin and binary skeleton. The thresholds to obtain the binary skeleton are determined by a root mean square (RMS) estimate of the noise. The nonmaxima suppression image is thresholded by the scaled mean value of the skeleton strength map of the brighter regions that is proportional to signal to noise ratio (SNR). For the proposed diffusion and the linear diffusion, the upper and the lower thresholds are determined as given below,

$T_{H}=s_{T} \times \frac{1}{M N} \sum_{x=1}^{M} \sum_{y=1}^{N}(S S M<0)_{x, y}, \quad T_{L}=T_{H} / 2$ 

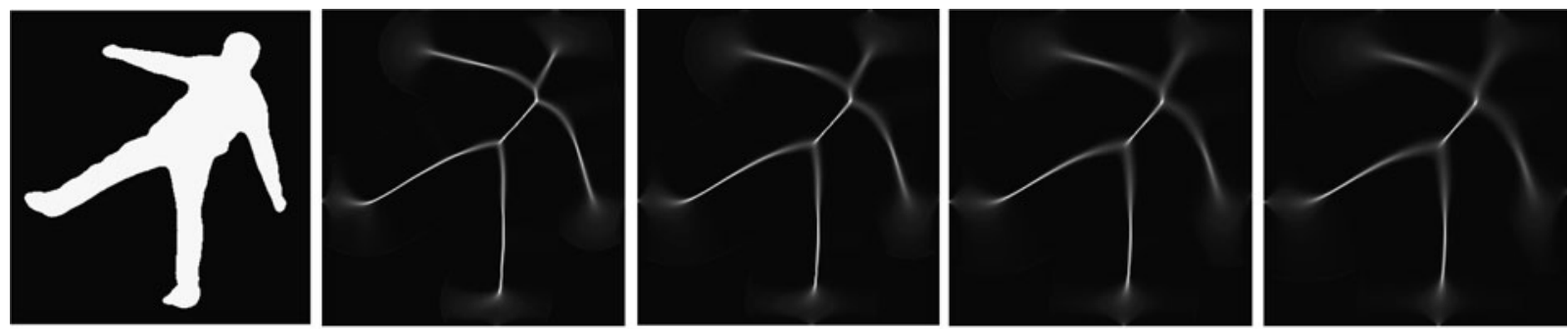

(a) Skeleton strength maps $(S S M<0)$ by linear diffusion (Lindeberg $(1998))$ with respect to increasing diffusion time $\left(t_{s}\right)$
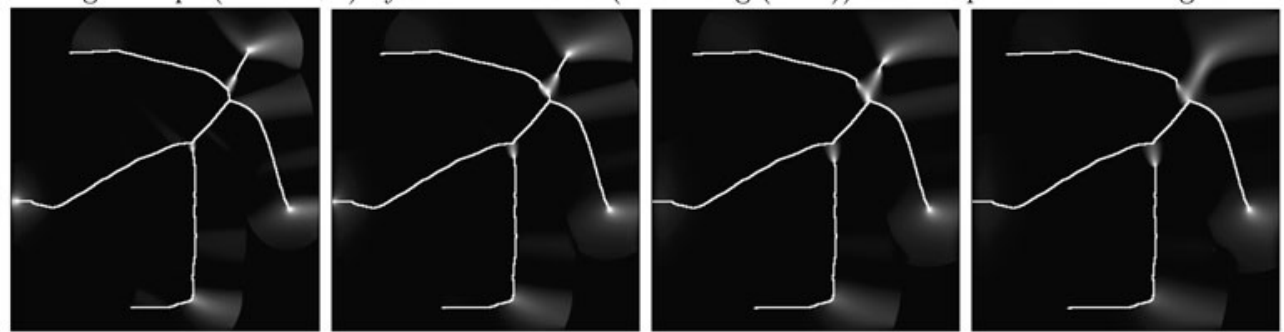

(b) Skeleton strength maps (SSAG) by AGVD (Yu and Bajaj (2004)) with respect to increasing diffusion time $\left(t_{s}\right)$
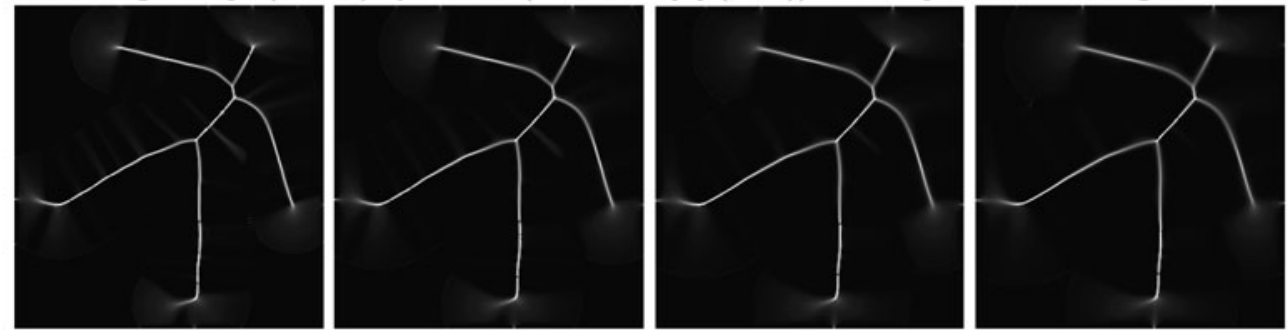

(c) Skeleton strength maps $(S S M<0)$ by proposed diffusion $(c=0.2)$ with respect to increasing diffusion time $\left(t_{s}\right)$
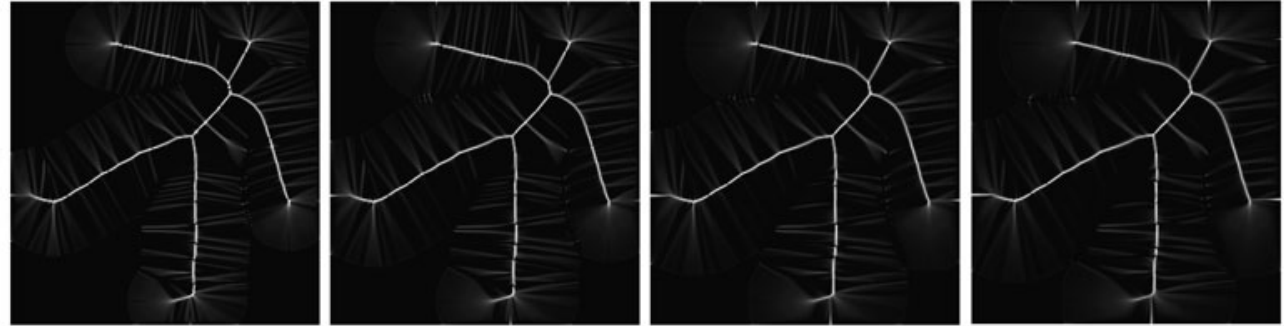

(d) Skeleton strength maps $(S S M<0)$ by proposed diffusion $(c=0)$ with respect to increasing diffusion time $\left(t_{s}\right)$

Fig. 5 Comparison of the proposed diffusion with linear diffusion and AGVD with respect to increasing diffusion time $\left(t_{s}\right)$ on binary image of a human shape of size $350 \times 335$. In Figs. $(\mathbf{a})-(\mathbf{d})$, the images from

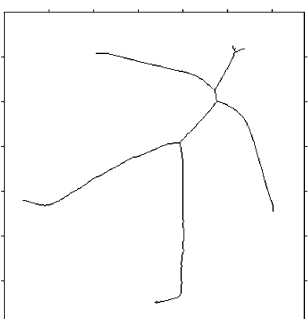

(a)

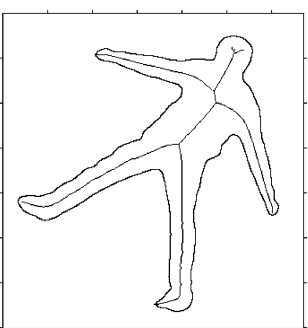

(b)
Fig. 6 Thin and binary skeleton obtained using the proposed diffusion with $c=0.2$ and $t_{s}=300$. (a) Binary skeleton after non-maxima suppression and hysteresis thresholding, (b) skeleton with shape boundary left to right show the skeleton strength maps for the brighter regions with respect to increasing diffusion times $t_{s}=300,500,700$ and 900 , respectively

where $M \times N$ is the size of the image $S S M<0$ and $s_{T}$ is a scale factor for threshold selection, which is a positive constant with value 9 in our experiments. The ratio between high, $T_{H}$, and low, $T_{L}$, thresholds is 2 . For the AGVD, the $S S M<0$ in Eq. (8) is replaced by SSAG and the same parameter values are used to determine the thresholds. These parameter values are determined experimentally to provide thresholds not higher than the maximum intensity values of the skeleton strength map for the brighter regions, but high enough to filter out noise.

Figure 7 shows skeleton extraction for the binary image of a human shape after adding salt and pepper noise. The first column in Fig. 7(a) shows the salt and pepper noise corrupted image with density $=0.3$. Figures $7(a), 7(b)$ and $7(\mathrm{c})$ 
Fig. 7 Experimentation with respect to salt and pepper noise $($ density $=0.3)$ in the binary image of size $350 \times 335$. For each method, the skeleton strength map for brighter regions, the binary skeleton and the binary skeleton superimposed to the original human shape are illustrated
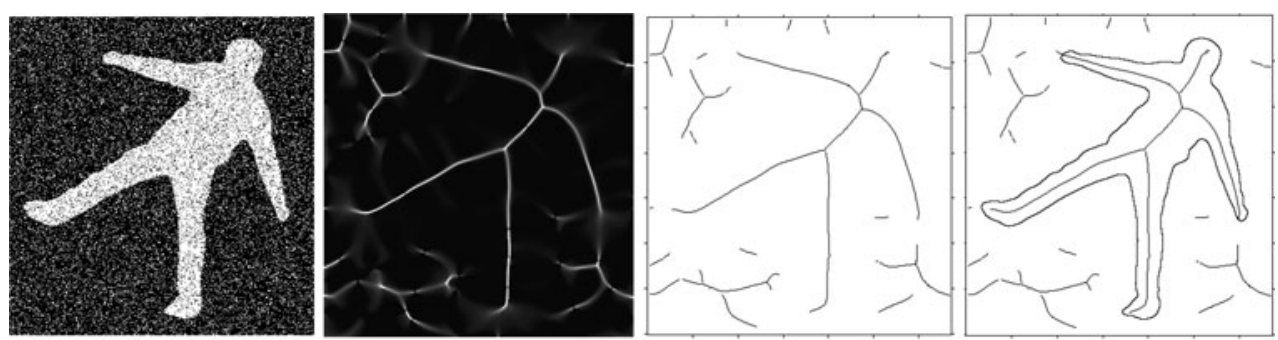

(a) Skeleton extraction with proposed diffusion $\left(c=0.2\right.$ and $\left.t_{s}=720\right)$
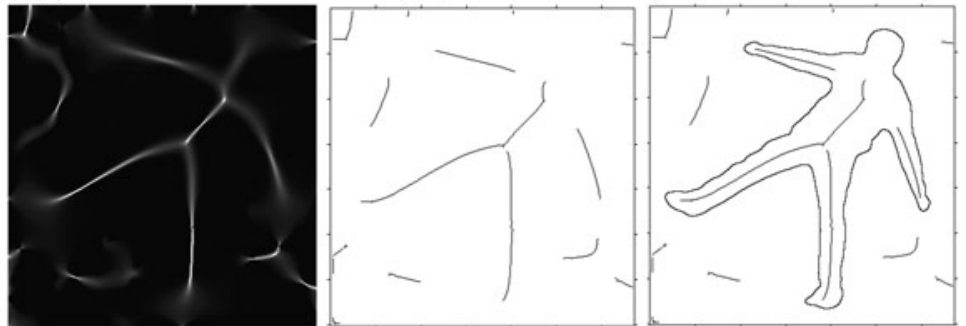

(b) Skeleton extraction with linear diffusion (Lindeberg (1998)) $\left(t_{s}=720\right)$
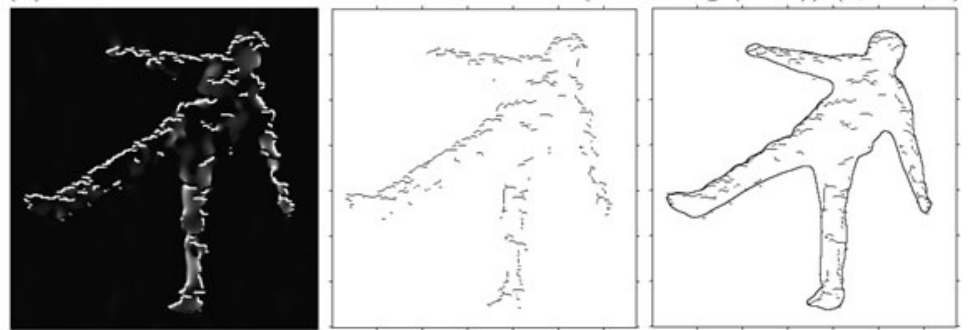

(c) Skeleton extraction with the AGVD (Yu and Bajaj (2004)) $\left(K=2\right.$ and $\left.t_{s}=300\right)$ show the skeletonization of the noisy image using the proposed diffusion $\left(t_{s}=720\right.$ and $\left.c=0.2\right)$, the linear diffusion $\left(t_{s}=720\right)$ and the AGVD $\left(K=2\right.$ and $\left.t_{s}=300\right)$ respectively. Here, $t_{s}$ values, for each method, are user defined and they are determined experimentally to deal with noisy instances of the image. For each method, the skeleton strength map for the brighter regions, the binary skeleton and the skeleton with the original shape of the subject is illustrated. Our proposed diffusion $(c=0.2)$ extracts the skeleton of the object without any dislocation and without missing any part of the subject. On the contrary, in linear diffusion it is observed that the skeleton features are not as strong as the skeleton features obtained by the proposed diffusion. For instance the skeleton of the shoulders, hands and feet are not detected. In linear diffusion, decreasing diffusion time $t_{s}$ would limit the ability of the method to remove the noise in the image. While increasing the diffusion time would cause more loss of skeleton features. Note that both the linear diffusion and the proposed diffusion methods detect skeletons on the background because the image is noisy. The AGVD cannot detect the skeleton of the shape, because AGVD uses gradient-based information which is very sensitive to the noisy conditions.

Figure 8 shows more results with the human silhouette corrupted with salt and pepper noise (density $=0.3$ ). Figure 8(a) shows the computed binary skeletons (four exper- iments with the same noise level) with the proposed diffusion, where the skeleton of the subject is consistently well extracted in all experiments (Fig. 8(a)). On the other hand, the linear diffusion keeps breaking the skeleton and losing some parts as shown in Fig. 8(b), and the AGVD fails to detect the skeleton in the noisy images (Fig. 8(c)).

Figure 9 shows skeleton extraction in the grey-scale image of human shape after adding Gaussian noise. The first column in Fig. 9(a) shows the Gaussian noise corrupted image with mean 0 and standard deviation 40 . In the original grey-scale image, the intensity value of the object region is 170 and the intensity value of background is 100 . Figures 9(a), 9(b) and 9(c) illustrate the skeleton extraction with the proposed diffusion ( $c=0.2$ and $t_{s}=720$ ), with the linear diffusion $\left(t_{s}=720\right)$ and with the AGVD $(K=2$ and $\left.t_{s}=720\right)$, respectively. For each method, we illustrate the skeleton strength map for the brighter regions, the binary skeleton and the skeleton with the original shape of the subject. While the proposed diffusion extracts well the skeleton of the subject, linear diffusion generates blurry skeleton features and some parts are then lost after thinning and thresholding (Fig. 9(b)). The AGVD cannot deal with the noise in the image, and so cannot extract the skeleton of the subject. All methods also extract skeletons in the background because of the noise. 
Fig. 8 More experimentation with respect to salt and pepper noise (density $=0.3$ ) in the human shape binary image of size $350 \times 335$
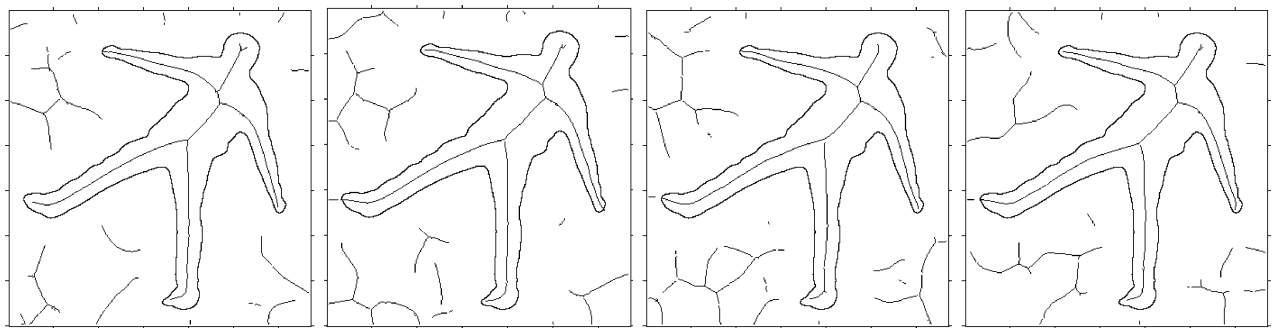

(a) Skeleton extraction with proposed diffusion $\left(c=0.2\right.$ and $\left.t_{s}=720\right)$
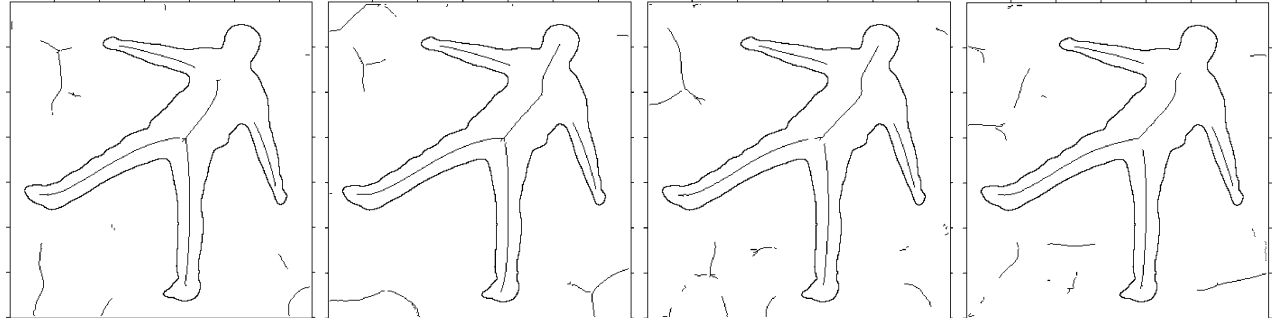

(b) Skeleton extraction with linear diffusion (Lindeberg (1998)) $\left(t_{s}=720\right)$
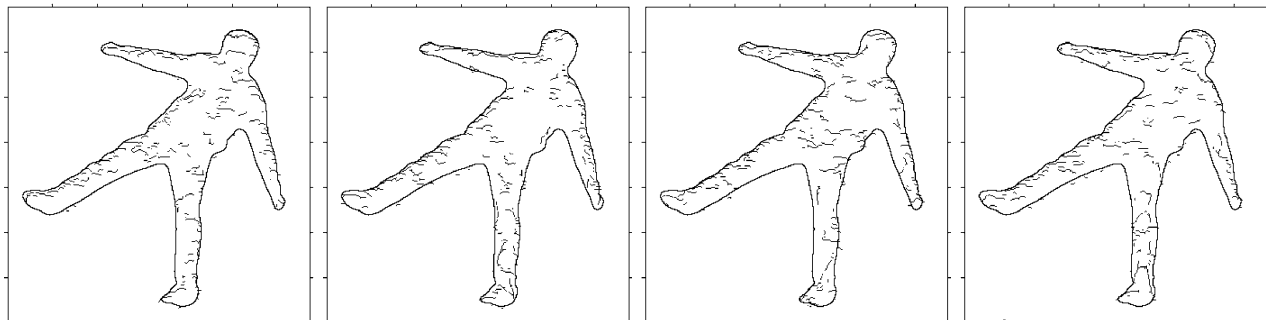

(c) Skeleton extraction with the AGVD (Yu and Bajaj (2004)) $\left(K=2\right.$ and $\left.t_{s}=300\right)$
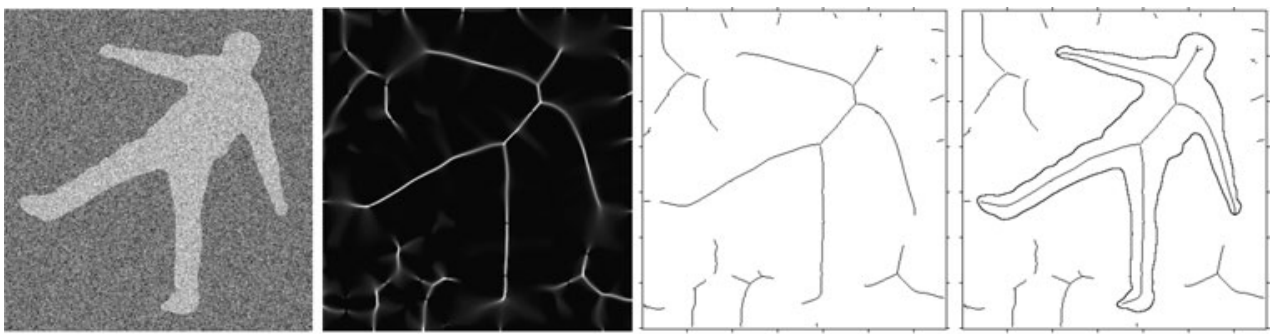

(a) Skeleton extraction with proposed diffusion $\left(c=0.2\right.$ and $\left.t_{s}=720\right)$

same image is illustrated
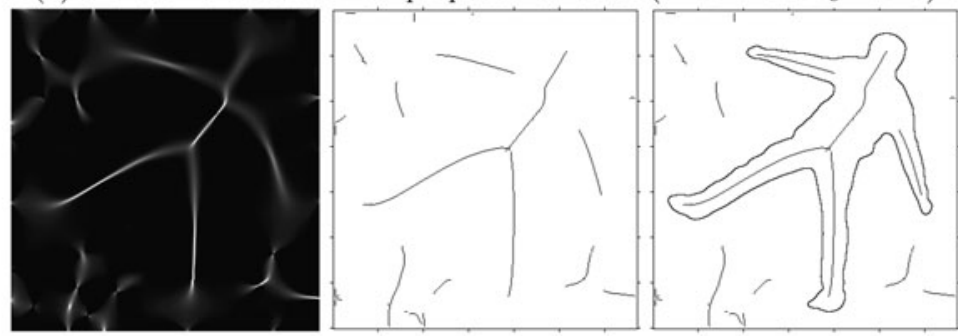

(b) Skeleton extraction with linear diffusion (Lindeberg (1998)) $\left(t_{s}=720\right)$
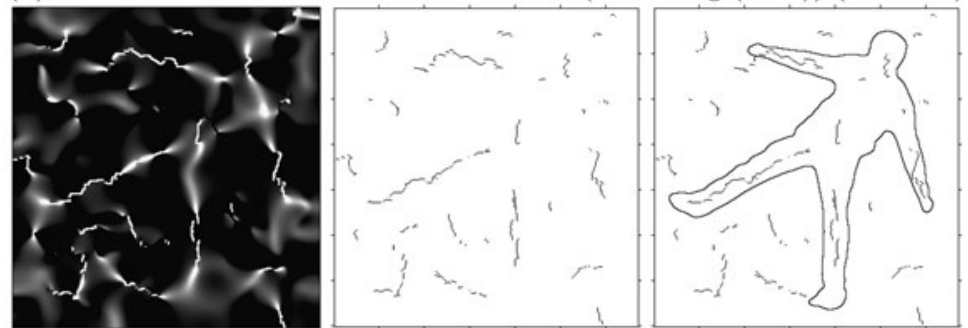

(c) Skeleton extraction with the AGVD (Yu and Bajaj (2004)) $\left(K=2\right.$ and $\left.t_{s}=720\right)$ 
Fig. 10 More experimentation with respect to Gaussian noise (mean $=0$ and standard deviation $=40$ ) in the human shape grey-scale image of size $350 \times 335$
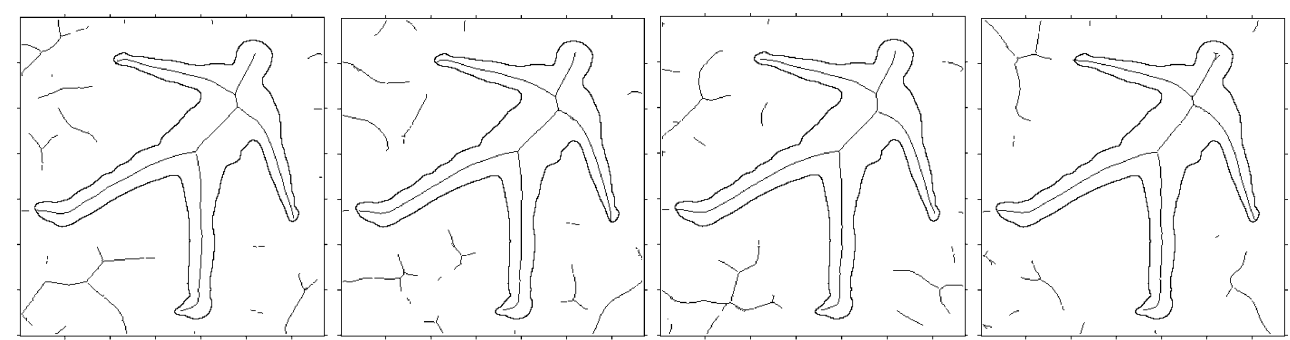

(a) Skeleton extraction with proposed diffusion $\left(c=0.2\right.$ and $\left.t_{s}=720\right)$

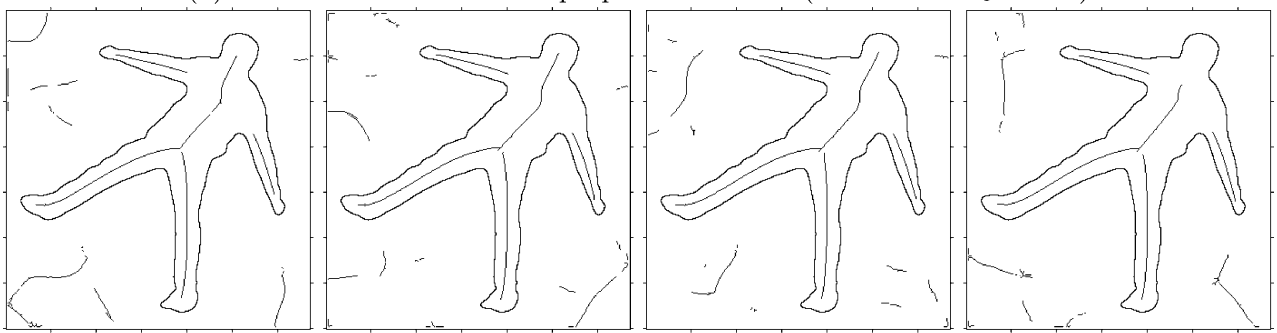

(b) Skeleton extraction with linear diffusion (Lindeberg (1998)) $\left(t_{s}=720\right)$
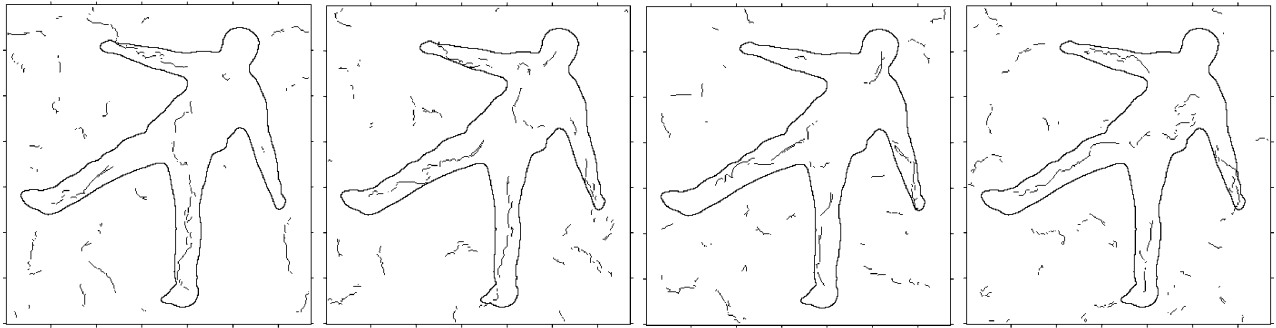

(c) Skeleton extraction with the AGVD (Yu and Bajaj (2004)) $\left(K=2\right.$ and $\left.t_{s}=720\right)$
Figure 10 shows four more skeletons extracted from the grey-scale image corrupted by Gaussian noise (same noise level: mean 0 and standard deviation 40). Note how the skeleton of the human shape is consistently well extracted in all experiments leading to a similar skeleton for our proposed approach (Fig. 10(a)). In comparison, linear diffusion and AGVD miss parts in the skeletons of the human shape (Fig. 10(b)). The skeleton obtained by the AGVD is very distorted.

\subsection{Quantitative Evaluations}

Based on the definition of the skeleton (Blum 1967), a skeleton point must be the center of a maximal disk/ball contained in the shape. Let $r(s)$ denotes the radius of the maximal disk $B(s, r(s))$ centered at a skeleton point $s$. The reconstruction of a shape using its skeleton is given by:

$R(S)=\bigcup_{s \in S} B(s, r(s))$

where $R(S)$ is the reconstructed shape and $S$ represents the skeleton. To evaluate the quality of the extracted skeleton, a reconstruction error rate $(R E R \%)$ between the reconstructed binary shape and the original binary shape is computed as follows:

$R E R \%=\frac{\operatorname{Area}(O)-\operatorname{Area}(R(S))}{\operatorname{Area}(O)} \times 100$ where $\operatorname{Area}(\cdot)$ denotes the area of the shape and it is measured in pixels. $O$ represents the image that contains the original binary shape and $R$ represents the image that contains the reconstructed binary shape. Note that similar evaluation method for skeleton extraction has been used by Shen et al. (2011) and by Hassouna and Farag (2005).

In quantitative evaluations, first we investigate the impact of diffusion time on the proposed diffusion, and compare with the linear diffusion and the AGVD on the noise free wave shape binary image (Sect. 3.2.1). Second, the effect of a Gaussian noise on the boundaries of a ring shape object in the binary image is assessed for the proposed diffusion, while comparing with the other diffusion methods (the linear diffusion and the AGVD), as well as with the popular and recent works (Bai et al. 2007 and Shen et al. 2011) in skeletonization of the binary images (Sect. 3.2.2). Third, we have experiments with the increasing salt and pepper noise in the binary image, where we measure both the performance of the proposed diffusion and the other diffusion methods which can achieve skeletonization without the need of any boundary initialization (Sect. 3.2.3). Finally, we evaluate the performances of the proposed diffusion and other diffusion methods on a grey-scale ring shape object which is corrupted by increasing Gaussian noise in the image (Sect. 3.2.4). The details of the evaluations are given in the following subsections. 


\subsubsection{Effect of Diffusion Time}

The diffusion time $t_{s}$ is an important parameter in diffusion process and in diffusion based skeletonization techniques. It affects the solution of the heat diffusion problem and consequently the result of the skeleton extraction. We investigate the impact of diffusion time on the proposed diffusion and compare with the linear diffusion and the AGVD in the original noise free binary image of the wave shape, where the size of the image is $562 \times 222$. This is a good synthetic shape to show both visually and quantitatively the performance of the diffusion methods in preserving the skeleton on the centre of the shape with increasing diffusion time. The evaluation is conducted at several diffusion times as follows:

1. First we extract the thin and binary skeleton of the wave shape in the image (thresholds are determined with Eq. (8)).

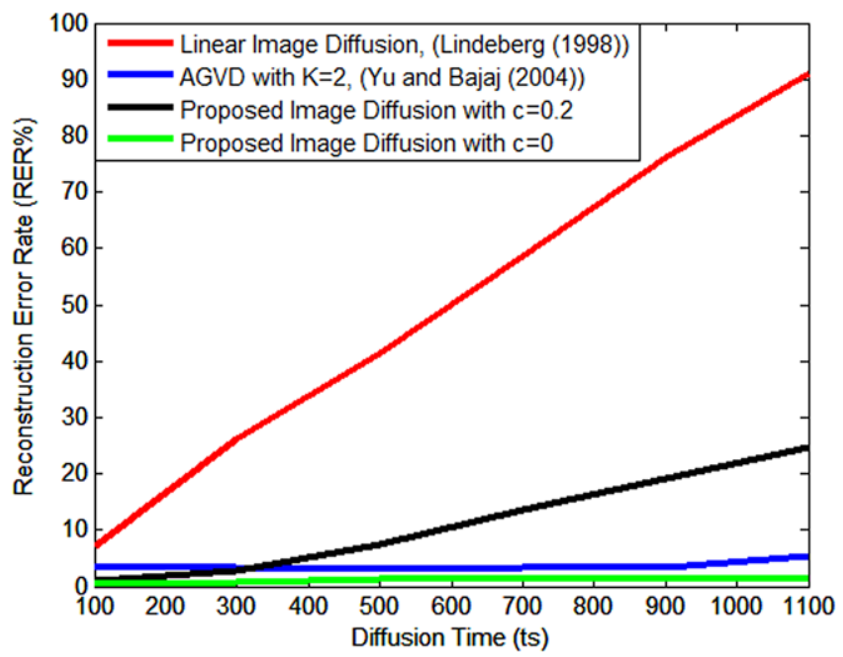

Fig. 11 Quantitative evaluation with respect to increasing diffusion time $\left(t_{s}\right)$ on a wave shape object
2. Then for each skeleton point we compute the radius of the maximal disk contained in the original shape.

3. And subsequently reconstruct the wave shape (Eq. (9)).

4. Finally, we compute the reconstruction error rate $(R E R \%)$ (Eq. (10)).

Figure 11 shows the RER\% with respect to an increasing diffusion time. In the proposed diffusion, two different conduction coefficients $(c=0$ and $c=0.2)$ are experimented. The selected diffusion times $\left(t_{s}\right)$ are: 100, 300, 500, 700, 900 and 1100 . The minimum diffusion time is $t_{s}=100$, since the linear diffusion and the proposed diffusion start to form reasonable medial functions after $t_{s}=100$ in this experiment. It is observed that as the diffusion time increases, the $R E R \%$ of the linear diffusion increases. Our results illustrates that the linear diffusion is very sensitive to diffusion time selection and it does not preserve the centeredness of the skeleton. The centeredness is not preserved and the skeleton is not accurate in the linear diffusion: the tangential diffusion causes curvature shrinkage which relocates or lose skeleton features. On the contrary, the proposed diffusion can preserve the centeredness of the skeleton better than the linear diffusion, since the proposed diffusion has slight contribution of the tangential diffusion. The proposed diffusion with $c=0.2$ achieves better than the linear diffusion but worse than the proposed diffusion with $c=0$. Choosing the conduction coefficient higher causes more contribution of the tangential diffusion that increases the reconstruction error. The AGVD with $K=2$ can also preserve the skeleton on the center of the shape with respect to increasing iteration. The proposed diffusion with $c=0$ achieves the smallest as well as the most stable reconstruction error rate (1-2 \%). This shows that choosing a small conduction coefficient removes the dependence on the diffusion time $t_{s}$ in skeleton extraction. Figures 12(a), 12(b) and 12(c) show some visual results for the proposed diffusion (a), the linear diffusion (b), and the AGVD (c) on the wave shape object with respect to diffusion times $t_{s}=200,500,900$ and 1100 .
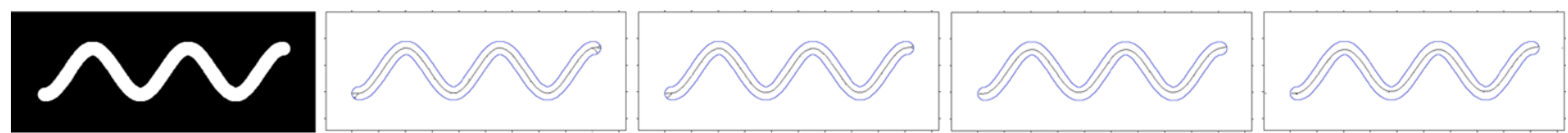

(a) Skeleton by the proposed diffusion with $c=0.2$ with respect to increasing diffusion time $\left(t_{s}=200,500,900,1100\right)$
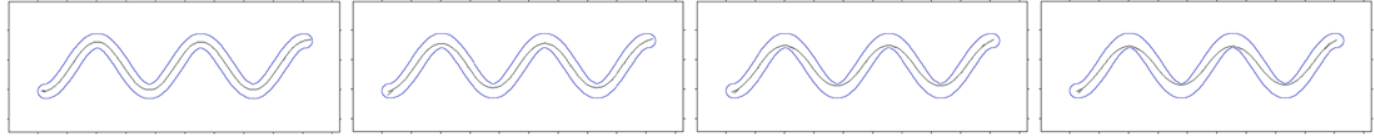

(b) Skeleton by the linear diffusion (Lindeberg (1998)) with respect to increasing diffusion time $\left(t_{s}=200,500,900,1100\right)$
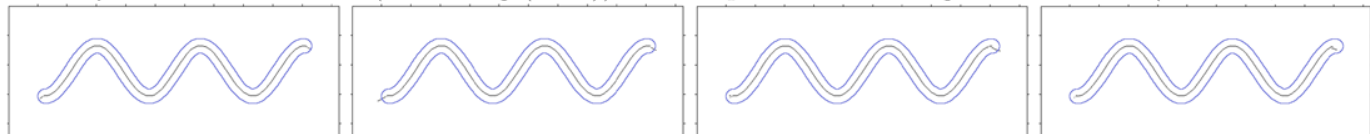

(c) Skeleton by the AGVD with $K=2$ (Yu and Bajaj (2004)) with respect to increasing diffusion time $\left(t_{s}=200,500,900,1100\right)$

Fig. 12 Experimentation on wave shape image of size $562 \times 222$ with respect to increasing diffusion time $\left(t_{s}\right)$ 
In Figs. 12(a), 12(b) and 12(c), the skeleton and the object shape (blue colour) are illustrated in the same image (at each diffusion time).

\subsubsection{Gaussian Noise on the Boundary}

We experiment the proposed image diffusion with respect to increasing Gaussian noise on the boundary of the ring shape object in a binary image of size $200 \times 200$. The proposed diffusion is compared to the most related diffusion models (i.e. linear diffusion and the AGVD) as well as to the popular and recent techniques (Bai et al. 2007 and Shen et al. 2011) in skeletonization of the binary images that need to initialize the boundaries of the shape. The ring shape binary image is a synthetic image. The skeleton of the ring shape should be a circle at the centre of the ring. If the skeleton (the circle) shape changes, it means the skeleton of the object is affected by the noise on the boundary. The amount of the sensitivity to the boundary noise can be measured with the $R E R \%$, and it is very simple to observe visually the changes of the circle shape.

The method, which is proposed by Bai et al. (2007), is based on using the Euclidean Distance (ED) map and the skeleton pruning using the Discrete Curve Evolution (DCE). This method will be denoted by ED + DCE in our evaluations. First, they select a skeleton seed point as a global maximum of the Euclidean distance map, and then the remainder of the skeleton points is decided by growing scheme. During this process, the redundant skeleton branches are eliminated by the DCE. In DCE based skeleton pruning, the object boundary is represented by a finite polygon, and the DCE eliminates the noisy points on the boundary by recursively removing polygon vertices with the smallest shape contribution. As a result, the DCE remains a subset of vertices that best represents the shape of the contour. The parameter $N$ is the number of vertices in the simplified boundary polygon and $N \geq 3$.

Recently, Shen et al. (2011) proposed another method for skeleton growing and pruning, called Bending Potential Ratio (BPR), in which the decision regarding whether a skeletal branch should be pruned or not is based on the context of the boundary segment that corresponds to the branch. The parameter $T$ is the measure of the BPR. When the value of the $T$ increases, there are fewer branches in the skeleton. The BPR pruning method can be applied to any input skeleton. In their work, they mainly use the Euclidean Distance (ED) transform for the skeleton growing, and combine with the BPR for skeleton pruning. This method is denoted by $\mathrm{ED}+\mathrm{BPR}$ in the evaluations.

The selected noise levels (standard deviations with mean $=0$ ) of the Gaussian noise are: 0.5, 1, 1.5, 2, 2.5, 3 and 3.5. The ring shape objects, after the Gaussian noise corruption on the boundaries, are shown in Fig. 14(a). The evaluation is conducted at each noise level as follows:
1. First the thin and binary skeleton of the noisy ring shape object in the image is extracted (thresholds for the diffusion models are determined with Eq. (8)),

2. Then for each skeleton point, the radius of the maximal disk contained in the original shape (noise free ring shape) is computed.

3. Next, the ring shape is reconstructed (Eq. (9)),

4. Finally the $R E R \%$ is computed (Eq. (10)).

Before the comparison to the other techniques, we test the proposed image diffusion with different conduction coefficient values (i.e. $c=0,0.1,0.2$ and 0.3 ), while keeping the diffusion time constant that is $t_{s}=500$. The conduction coefficient controls the amount of contribution of the tangential diffusion. The performance of the proposed diffusion with different conduction coefficient values are shown in Fig. 13(a). The graphs include error bars representing the mean and standard deviation computed after repeating the experiment five times. It is observed that as the conduction coefficient increases, it shifts the skeleton offset from the ideal center, when there is no noise or small amount of noise (i.e. $<1.5$ ) on the boundary. On the other hand, when there is a higher amount of noise (i.e. $\geq 1.5$ ), it is better to have a conduction coefficient with a higher value (i.e. $c=0.2$ or $c=0.3$ ) since it can remove the noise and preserve the skeleton features better on the ideal center.

Figure 13(b) compares the RER\% of the proposed image diffusion and of the other methods with respect to the increasing standard deviation of the Gaussian noise on the boundary. The conduction coefficient is $c=0.2$ for our method and the diffusion time is $t_{s}=500$. For the linear diffusion, the diffusion time is $t_{s}=300$. In AGVD, $K=1$ and the diffusion time is $t_{s}=300$. In ED + DCE based skeleton pruning, $N=3$. The parameter $N$ is the number of vertices in the simplified boundary polygon and it cannot be less than 3, which means we should observe 3 skeleton branches on the skeleton of the ring. In ED + BPR based skeleton pruning, $T=10$. The parameter $T$ is the threshold for the BPR and it is determined experimentally to remove all the branches on the skeleton of the ring shape. Note that all of the parameters in this evaluation are determined experimentally to achieve the best results for each method.

The ED + DCE and the ED + BPR methods perform similarly and as the noise increases on the boundary the RER\% increases for these methods. Indeed, they both use Euclidean distance map as a medial function that is sensitive to the boundary deformations. The distance transform is sensitive to the small perturbations of the boundary, since each value on shape is assigned according to the single boundary point (nearest one). Although the DCE and the BPR can be effective skeleton pruning methods, the centeredness of the skeleton is determined by the distance transform. Figure 14(e) and (f) show some of the results for the ED + DCE and for the ED + BPR, respectively. The skeleton and the original 


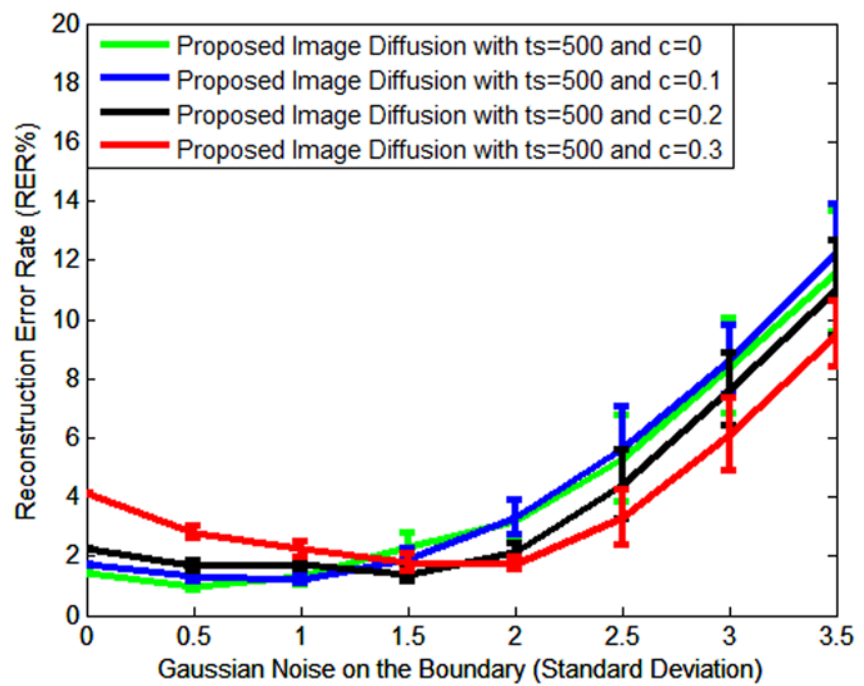

(a)

Fig. 13 Quantitative evaluation with respect to increasing Gaussian noise on the boundary. (a) The proposed image diffusion with different conduction coefficient values (i.e. $c=0,0.1,0.2$ and 0.3 ), while

object shape (in blue colour) are shown in the same image. The skeletons (circle shapes) are distorted after the standard deviation is 1 . The ED + DCE and the ED + BPR performs better than other techniques when the standard deviation is inferior to 1 . However when the noise increases (standard deviation superior to 1), our proposed diffusion performs better than these two methods, since they are sensitive to noise.

The proposed diffusion is robust in extracting a consistent skeleton when the standard deviation is inferior to 2 . For higher level of noise, the skeleton starts to dislocate. Although the skeleton dislocates with increasing noise, the skeleton shape is a smooth circle. When there is no noise or low noise, i.e. standard deviation: $0,0.5$ and 1 , the proposed diffusion shifts the skeleton and cause around $2 \%$ error, since we have a small amount of contribution of the tangential diffusion, i.e. $c=0.2$, in this experiment. The proposed diffusion also outperforms the linear diffusion with $t_{s}=300$ until standard deviation is equal to 2 . The proposed diffusion can remove noise on the boundary and preserve skeleton location up to a certain level of noise. On the other hand, the linear diffusion can remove noise but also relocate skeleton and cause reconstruction error. This is a consequence of the full contribution of the tangential diffusion in the linear diffusion. In addition, choosing the diffusion time less than $t_{s}=300$ (in this experiment) may not be enough to remove noise and generate a medial function. Figures 14(b) and (c) illustrate visual results for the proposed diffusion ( $c=0.2$ and $\left.t_{s}=500\right)$ and visual results for the linear diffusion $\left(t_{s}=300\right)$, respectively. It is observed that both methods can keep the circle shape skeleton smooth at different noise levels.

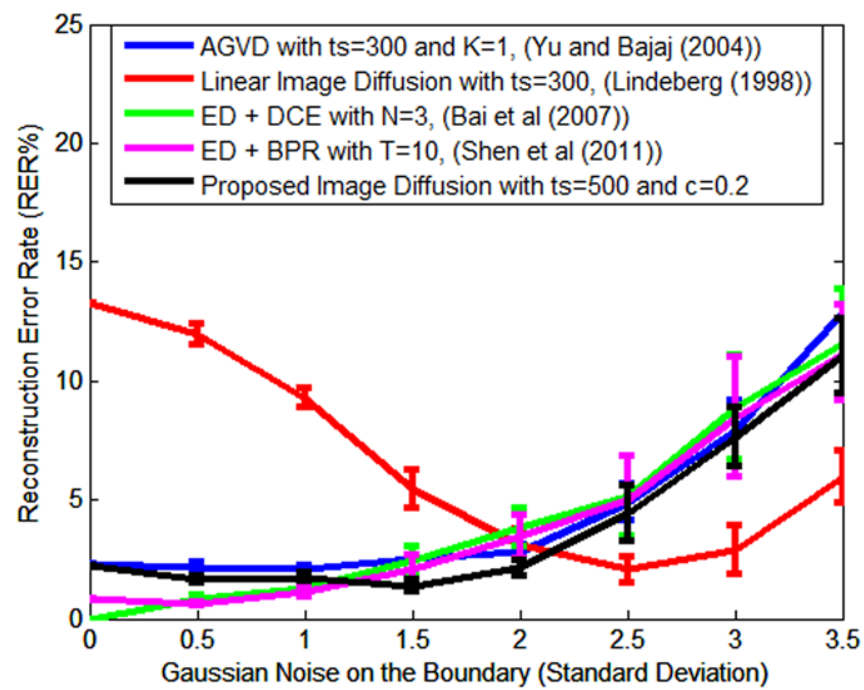

(b)

keeping the diffusion time constant that is $t_{s}=500$. (b) The proposed image diffusion is compared to the other methods

The proposed image diffusion also performs better than the AGVD at all noise levels. The AGVD is more sensitive to the noise on the boundary, and the skeleton is broken at some noise levels as shown in Fig. 14(d).

\subsubsection{Salt and Pepper Noise in the Image}

The proposed image diffusion, the linear diffusion and the AGVD are assessed with respect to an increasing salt and pepper noise in the binary image. Note that we cannot assess the ED + DCE and the ED + BPR methods in this case, because these methods need to know the boundary of the shape. The selected noise levels (densities) are: 0.1, 0.2, 0.3, $0.4,0.5$ and 0.6. The salt and pepper noise corrupted ring shape binary images are shown in Fig. 16(a). The evaluation is conducted at each noise level as follows:

1. The skeletons in the noisy image are extracted. In this case, there are also some skeletons detected in the background because of the noise: Figs. 16(b), (c) and (d) show detected skeletons using the proposed diffusion, the linear diffusion and the AGVD, respectively.

2. Then for each skeleton point inside the original ring shape object (which is shown with blue colour in the skeleton images), we compute the radius of the maximal disk contained in the original shape (in blue colour).

3. The ring shape is then reconstructed (Eq. (9)),

4. The RER\% is computed (Eq. (10)).

Figure 15(a) shows the performances of the algorithms. The graphs include error bars representing the mean and standard deviation computed after repeating the experiment five 

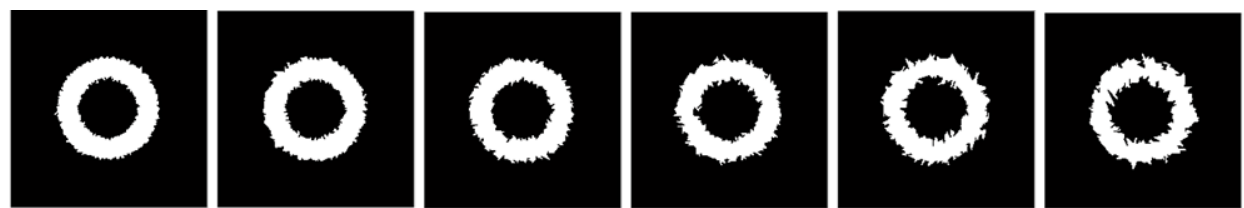

(a) Ring shape objects with increasing Gaussian noise on the boundary. Standard deviations: 1, 1.5, 2, 2.5, 3, 3.5.
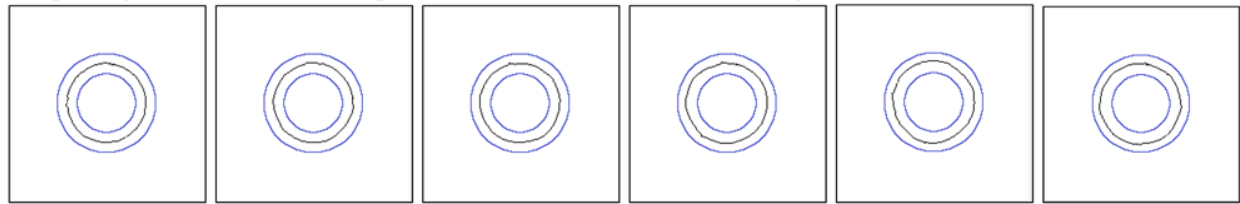

(b) The proposed image diffusion with $c=0.2$ and $t_{s}=500$ based skeletonization

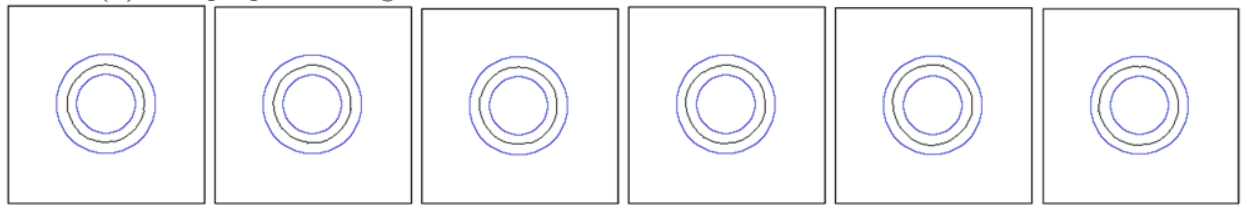

(c) Linear image diffusion with $t_{s}=300$ (Lindeberg (1998)) based skeletonization

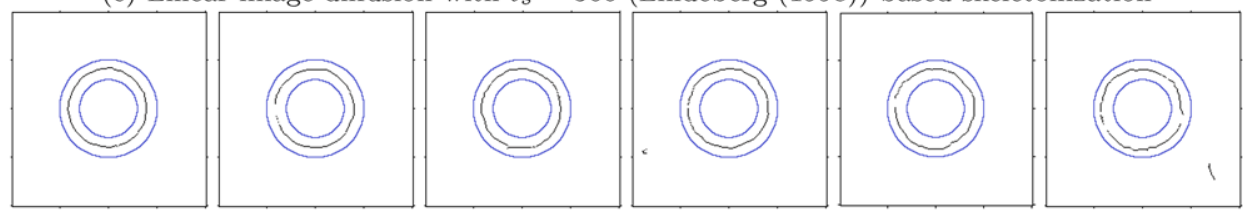

(d) The AGVD with $K=1$ and $t_{s}=300$ (Yu and Bajaj (2004)) based skeletonization

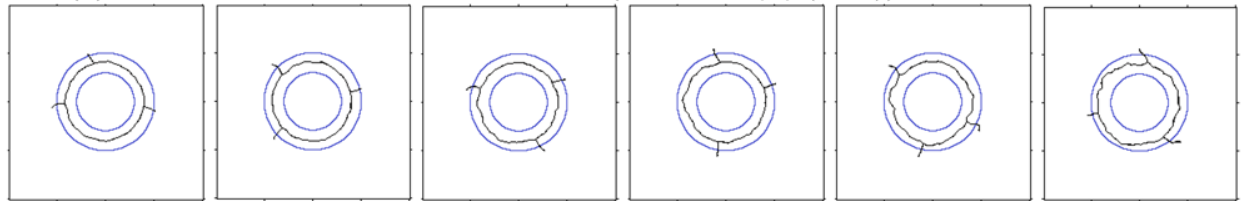

(e) The ED+DCE with $\mathrm{N}=3$ (Bai et al. (2007)) based skeletonization

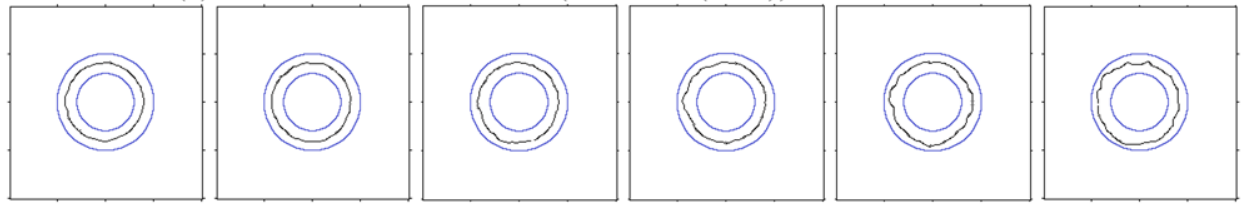

(f) The ED+BPR with $\mathrm{T}=10$ (Shen et al. (2011)) based skeletonization

Fig. 14 Skeletonization of the ring shape image of size $200 \times 200$ with respect to increasing Gaussian noise on the boundary

times. The linear diffusion is computed with two diffusion times $t_{s}=300$ and with $t_{s}=500$. Our proposed diffusion ( $\left.c=0.2, t_{s}=500\right)$ outperforms the linear diffusion (with $t_{s}=300$ and $\left.t_{s}=500\right)$ and the AGVD ( $K=2$ and $\left.t_{s}=300\right)$. The proposed diffusion can preserve skeleton location up to high noise levels. The AGVD cannot handle the salt and pepper noise in the image and extracts very distorted skeletons. As the noise increases, the RER\% increases for the AGVD. The linear diffusion can remove noise in the image but does not preserve skeleton locations at the center of the shape, which causes reconstruction error.

\subsubsection{Gaussian Noise in the Image}

Finally, we evaluated the performance of the linear diffusion, the AGVD and the proposed diffusion on a grey-scale ring shape object with respect to increasing Gaussian noise in the image. The selected noise levels (standard deviations with mean $=0$ ) are: $10,20,30,40,50$ and 60. The Gaussian noise corrupted grey-scale images are illustrated in Fig. 17(a). The evaluation is conducted at each noise level as follows:

1. First we extract the skeletons in the noisy image. Figures 17(b), (c) and (d) show detected skeletons respectively using the proposed diffusion, the linear diffusion and the AGVD at each noise level.

2. Then for each skeleton point inside the original ring shape object (shown with blue colour in the skeleton images), we compute the radius of the maximal disk contained in the original shape (in blue colour).

3. The ring shape is then reconstructed (Eq. (9)).

4. Finally the $R E R \%$ is computed (Eq. (10)). 


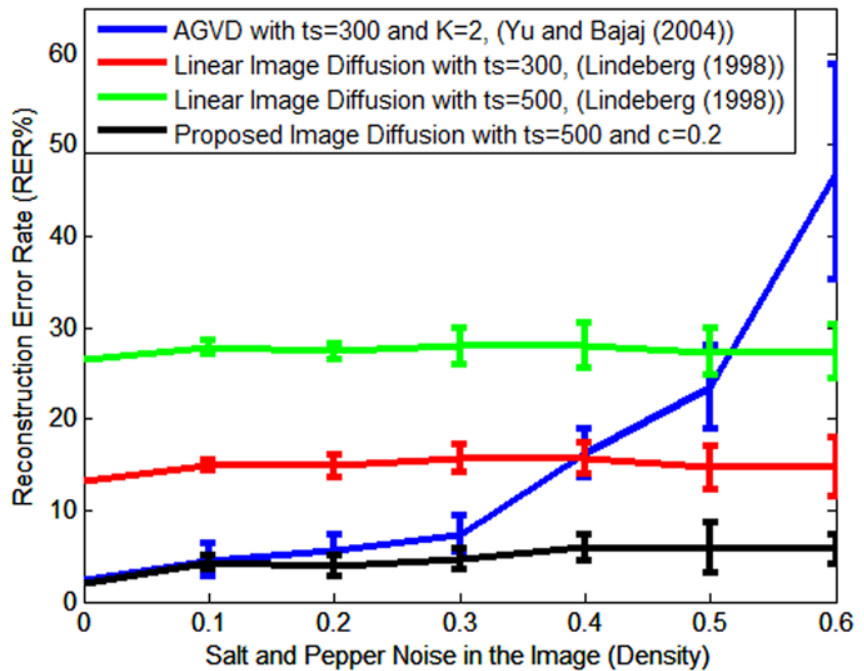

(a)

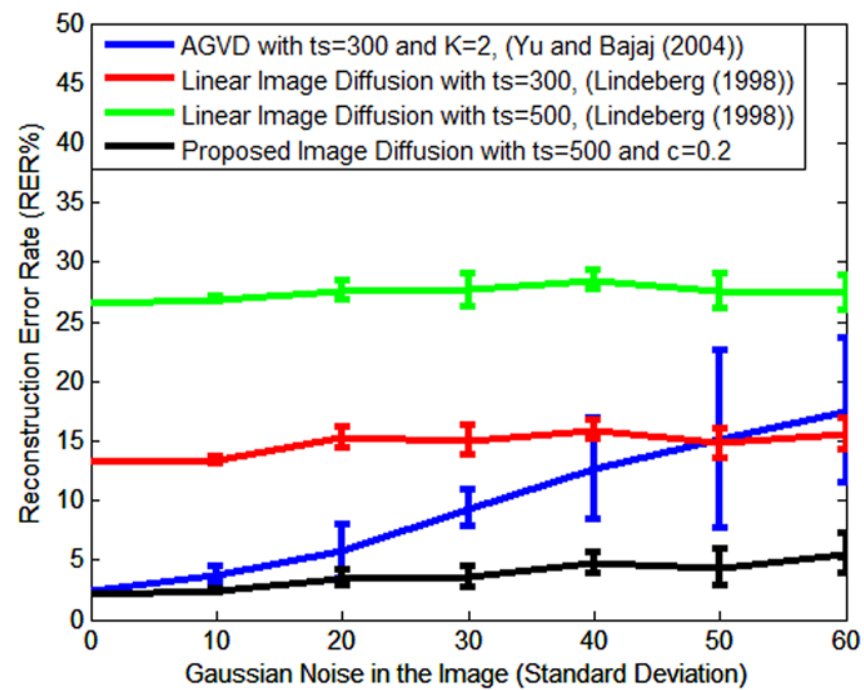

(b)

Fig. 15 (a) Quantitative evaluations with different noise types in images. (a) Evaluation with respect to increasing salt and pepper noise in the binary image. (b) Evaluation with respect to increasing Gaussian noise in the grey-scale image
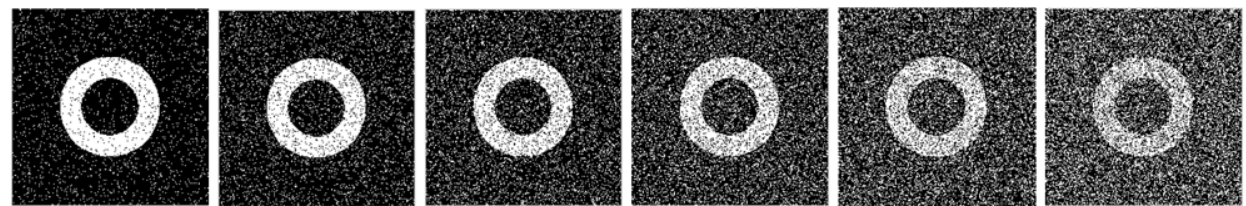

(a) Ring shape object with increasing salt and pepper noise in the binary image. Densities: $0.1,0.2,0.3,0.4,0.5,0.6$.

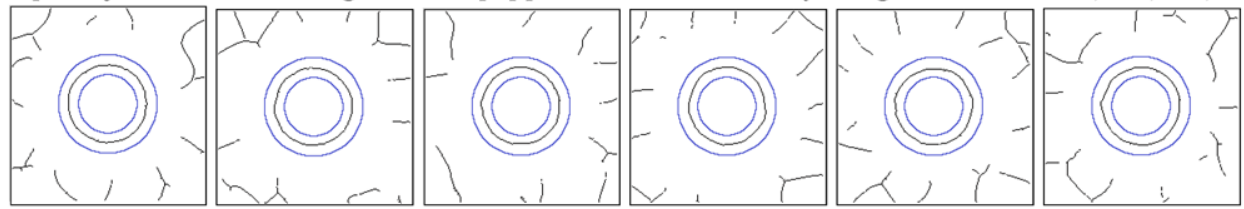

(b) The Proposed Diffusion with $c=0.2$ and $t_{s}=300$ based skeletonization

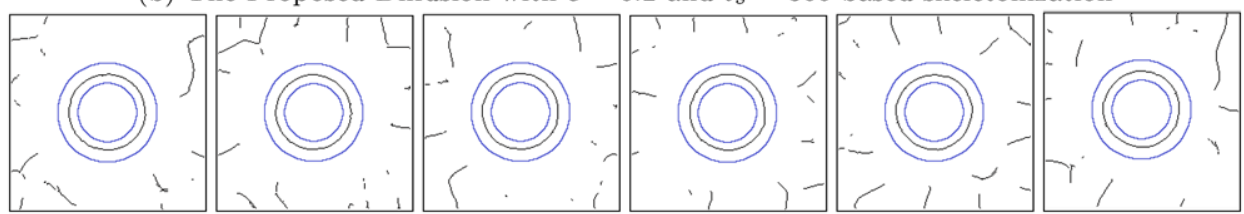

(c) Linear Diffusion with $t_{s}=300$ (Lindeberg (1998)) based skeletonization

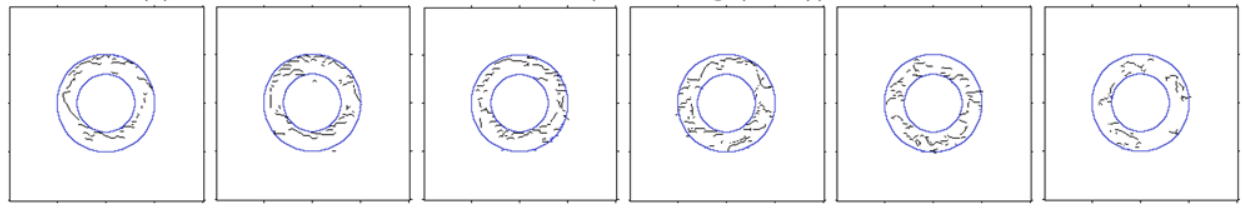

(d) The AGVD with $K=2$ and $t_{s}=300$ (Yu and Bajaj (2004)) based skeletonization

Fig. 16 Skeletonization of the ring shape image of size $200 \times 200$ with respect to increasing salt and pepper noise in the image

Figure 15(b) shows the RER\% with respect to increasing Gaussian noise in the image. The graphs include error bars representing the mean and standard deviation computed after repeating the experiment five times. The linear diffusion has parameters $t_{s}=300$ and $t_{s}=500$, our diffusion is experimented with $t_{s}=500$ and $c=0.2$, and the AGVD is experimented with $t_{s}=300$ and $K=2$. The results with the Gaussian noise are similar to the results obtained with the salt and pepper noise. The proposed diffusion outperforms the linear diffusions $\left(t_{s}=300\right.$ and $\left.t_{s}=500\right)$ and the AGVD. Indeed the linear diffusion can remove noise in the image but does not preserve skeleton features at the center. The 

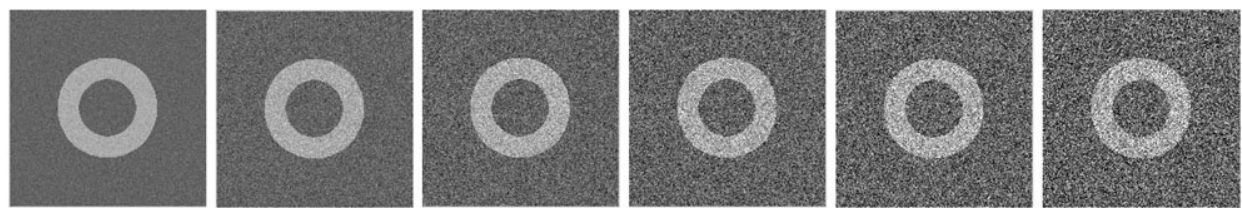

(a) Ring shape object with Gaussian noise in the grey-scale image. Standard deviations: 10, 20, 30, 40, 50, 60.

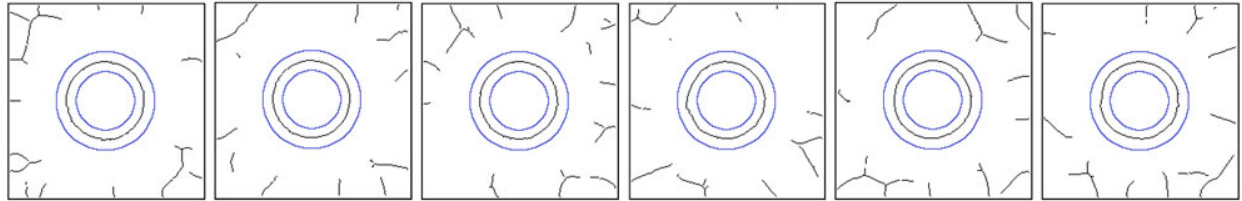

(b) The Proposed Diffusion with $c=0.2$ and $t_{s}=300$ based skeletonization

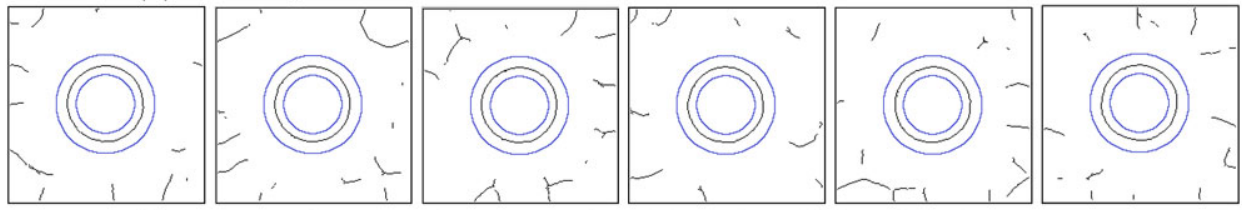

(c) Linear Diffusion with $t_{s}=300$ (Lindeberg (1998)) based skeletonization

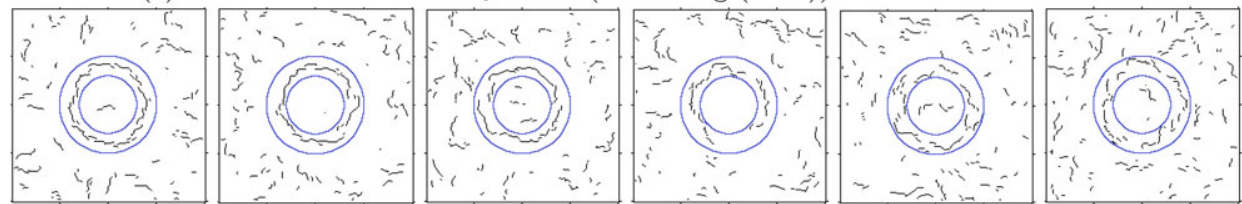

(d) The AGVD with $K=2$ and $t_{s}=300$ (Yu and Bajaj (2004)) based skeletonization

Fig. 17 Skeletonization of the ring shape image of size $200 \times 200$ with respect to increasing Gaussian noise in the image

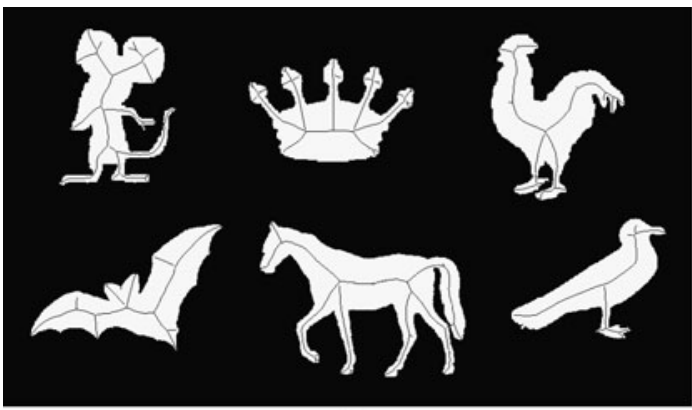

(a)

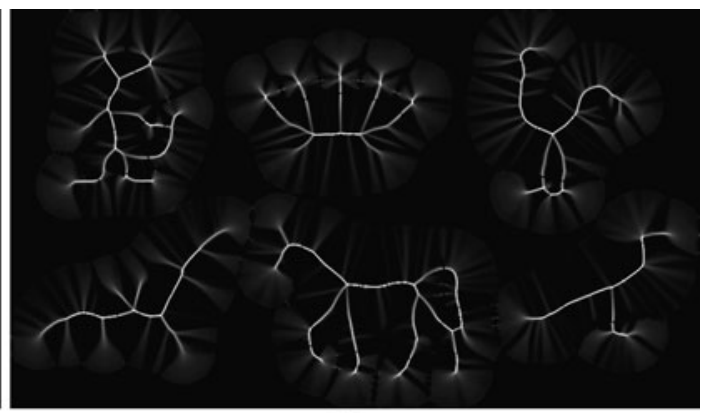

(b)

Fig. 18 Skeletonization of shapes from the MPEG-7 shape dataset in the same image

tangential diffusion relocates the curvature maxima and violates centeredness of the skeleton. On the other hand, the AGVD uses a gradient-based algorithm, which is sensitive to the noisy conditions.

\subsection{Multiple Objects in a Binary Image}

Figure 18 shows skeletonization of multiple objects in a binary image of size $509 \times 880$, using the proposed diffusion with $c=0.1$ and $t_{s}=250$. These shapes are from MPEG-7 dataset (Latecki et al. 2000). Figure 18(b) is the $S S M<0$, and Fig. 18(a) is the skeletons with the objects in the same image. It is observed that the proposed algorithm can detect skeletons of the multiple objects in the image.

\subsection{Gray-Scale Images}

Experimental results also show that our algorithm can extract skeletons in gray-scale images. Figure 19(a) shows a human brain image of size $413 \times 404$. Figure 19 (b) shows the $S S M<0$ that is computed after proposed anisotropic diffusion with parameter values $c=0.2$ and $t_{s}=60$. The $S S M<0$ represents the skeleton strength map of the brighter regions in the image. The binary skeleton computed from the $S S M<0$ is shown in Fig. 19(c). The $S S M>0$ represents the skeleton strength map of the darker regions is shown in Fig. 19(d). The binary skeleton obtained from the $S S M>0$ is illustrated in Fig. 19(e). It is observed that skeletons of the brighter and the darker regions are detected. 
Fig. 19 Skeletonization of gray-scale brain image of size $413 \times 404$. (a) The human brain, (b) skeleton strength map for brighter regions $(S S M<0)$, (c) binary skeleton for $S S M<0$, (d) skeleton strength map for darker regions $(S S M>0)$,

(e) binary skeleton for $S S M>0$

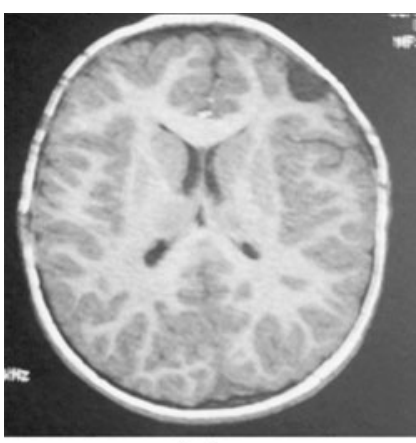

(a)

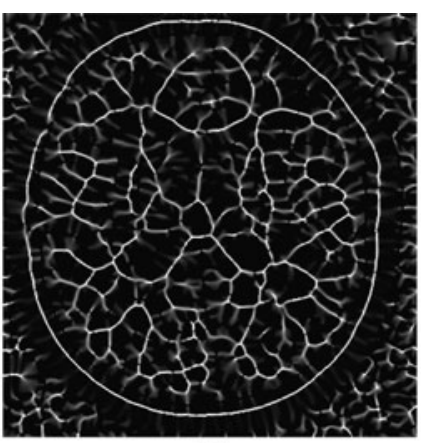

(b)

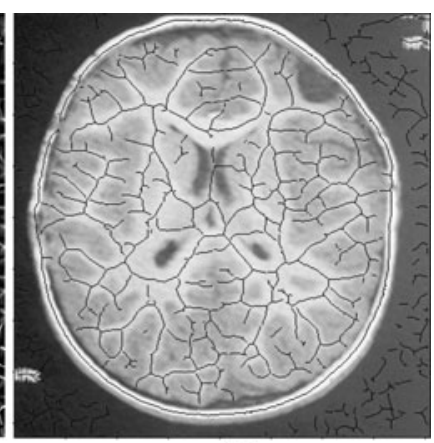

(c)

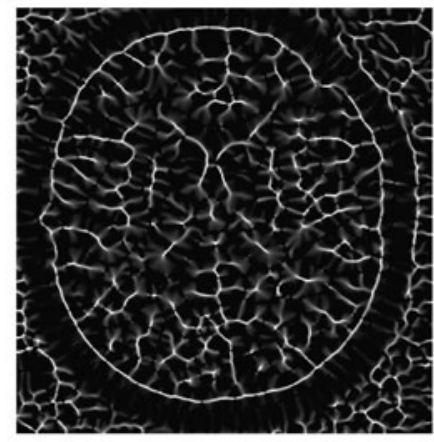

(d)

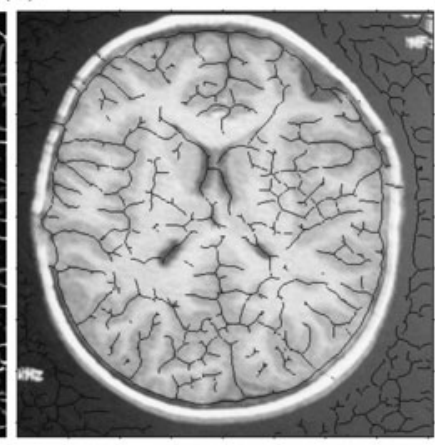

(e)
Figure 20 shows skeleton extraction in a gray-scale image of size $245 \times 829$. In this image (see Fig. 20(a)), there is a cheetah that is lying down, as well as tree branches and leaves in the background. Figure 20(b) is the $S S M<0$ (for brighter regions) that is computed after proposed diffusion with parameter values $c=0.1$ and $t_{s}=60$. Figure 20(c) shows the binary skeleton that is obtained from the $S S M<0$. Here, skeleton curves are illustrated with a black color, since it is easier to see and realize them on brighter regions. It is observed that skeletons of the brighter regions are detected. Especially, one can see the skeleton curves of the brighter regions on the body, head, tail and limbs of the cheetah. Skeleton curves around black spots and around nose and eyes are extracted. Skeletons of the brighter regions in the background between tree branches and leaves are detected. The skeleton strength map of the darker regions, $S S M>0$, is shown in Fig. 20(d). The binary skeleton computed from the $S S M>0$ is shown in Fig. 20(e). Here, skeleton curves are illustrated with a white color to realize them better on darker regions. Skeletons of the darker regions, on the body, head, tail and limbs of the cheetah, are extracted. Skeletons of the tree branches and leaves, which are darker regions in the background, are well extracted.

Figure 21 also shows skeletonization of a gray-scale image of size $431 \times 348$. This is an image of a traffic sign on the street, which represents the presence of a school (see Fig. 21(a)). There is also grass in the background. Figure 21(b) shows the $S S M>0$ (for darker regions) that is computed after proposed diffusion with parameter values $c=0.1$ and $t_{s}=70$. Figure 21(c) is the binary skeleton that is obtained from the $S S M>0$. Here, skeleton curves are illustrated with a white colour. It is observed that skeletons of the darker regions are detected in the image. Especially, one can see the skeleton curves of human shapes on the traffic sign. There are also skeleton curves detected on the traffic sign because of shadows and changing illumination conditions. Skeleton of the grass is also detected, which represents the textured nature of the grass. The skeleton strength map of the brighter regions, $S S M<0$, is illustrated in Fig. 21(d). The binary skeleton computed from the $S S M<0$ is in Fig. 21(e). Here, skeleton curves are shown with a black colour to observe them better on bright regions. It is seen that skeletons of the brighter regions are extracted on the traffic sign and in the background as well.

\section{Conclusions and Future Work}

We have presented a novel and effective skeleton extraction algorithm based on the anisotropic heat flow analogy. A medial function of high quality is computed with the proposed anisotropic diffusion. Then, the skeleton strength map $(S S M)$ is defined with the mean curvature measure. Finally, non-maxima suppression and hysteresis thresholding is applied to obtain a thin and binary skeleton. The proposed anisotropic image diffusion provides high quality skeleton features and preserves the skeleton features at the centerline of the shape, while denoising the image and the object boundaries. The proposed diffusion outperforms the most 
Fig. 20 Skeletonization of a gray-scale cheetah image of size $245 \times 829$. Skeletons of the brighter and the darker regions are detected

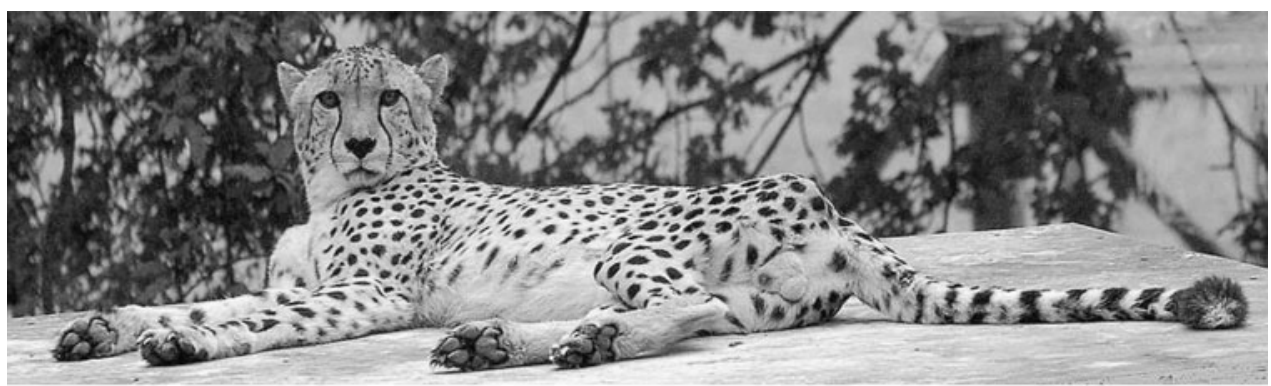

(a) The cheetah image

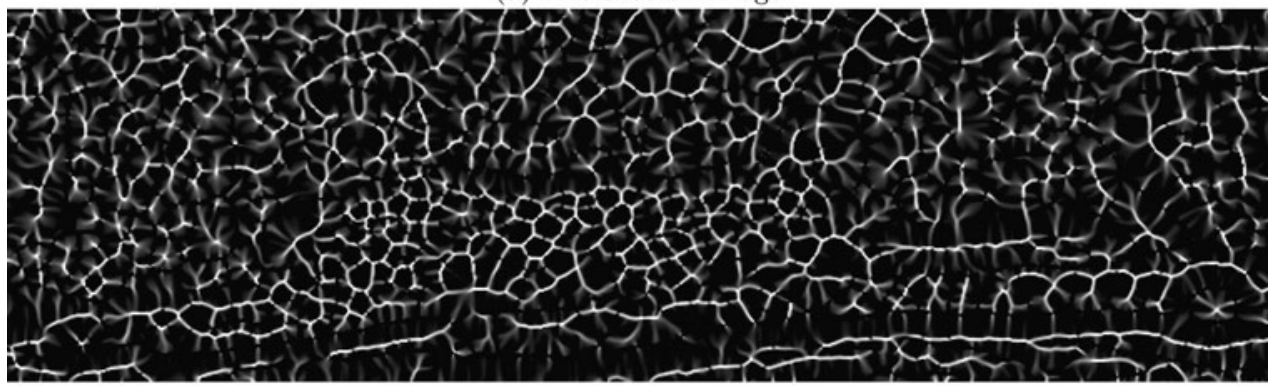

(b) Skeleton strength map for brighter regions $(S S M<0)$

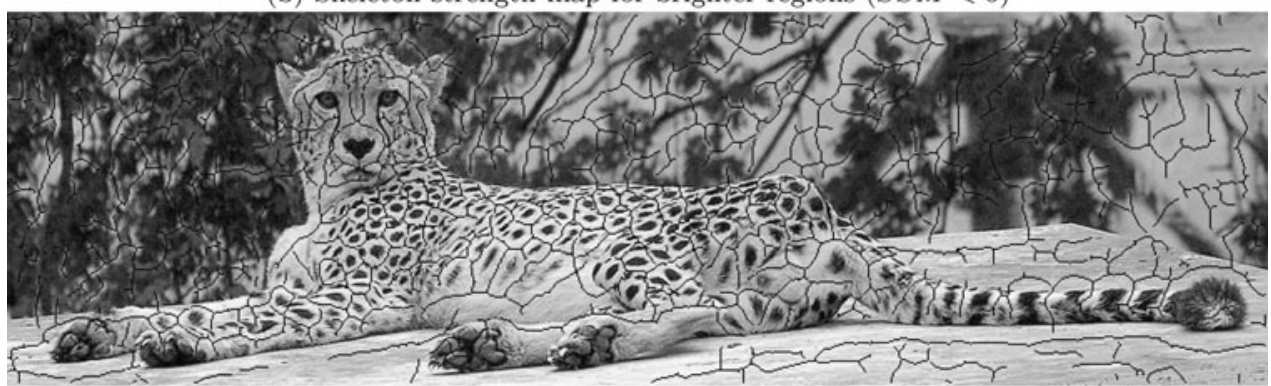

(c) Binary skeleton for brighter regions $(S S M<0)$

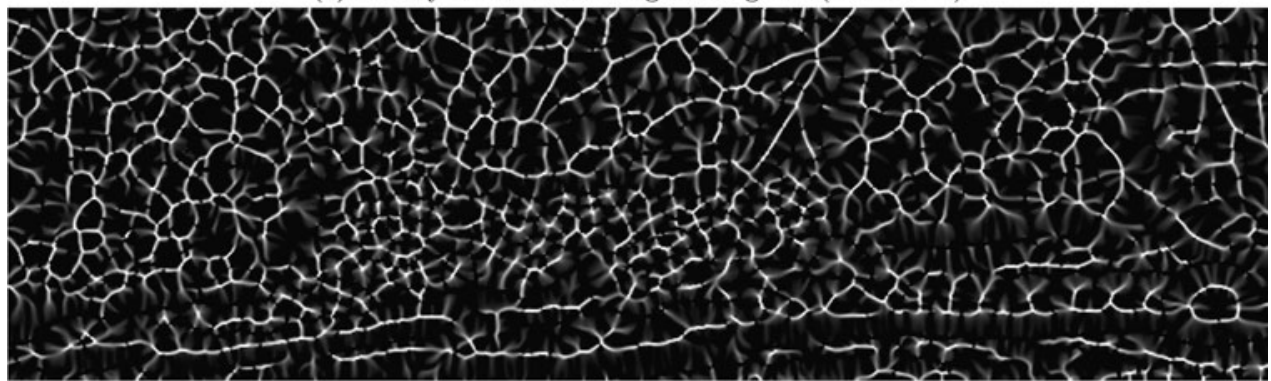

(d) Skeleton strength map for darker regions $(S S M>0)$

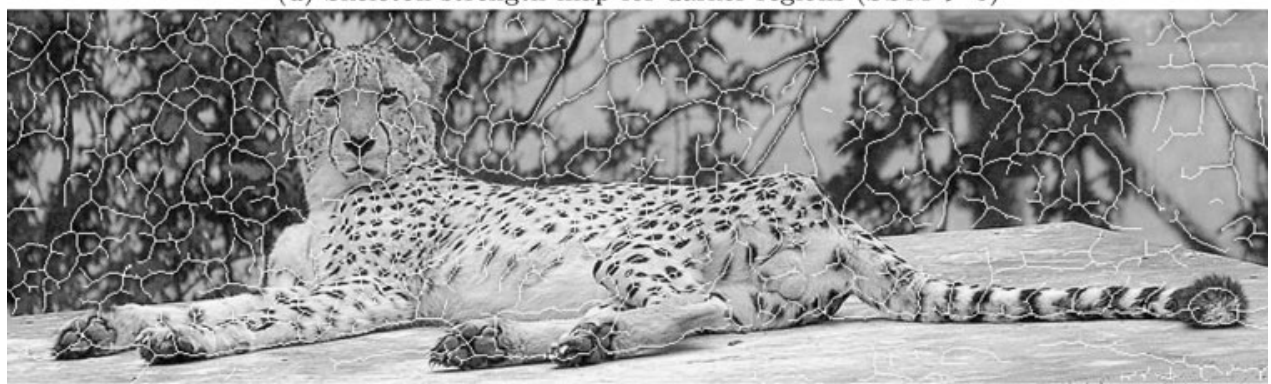

(e) Binary skeleton for darker regions $(S S M>0)$

related diffusion based methods and the other popular methods in skeleton extraction especially in noisy conditions. Results also show that our technique can be applied to both bi- nary and gray-scale images without the need of any shape extraction, boundary initialization or skeleton pruning. In future work, we will focus on developing mechanisms for 


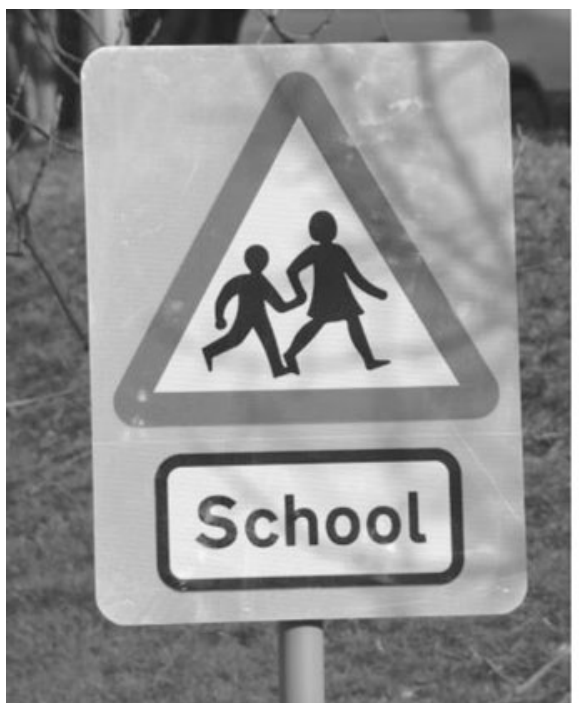

(a)

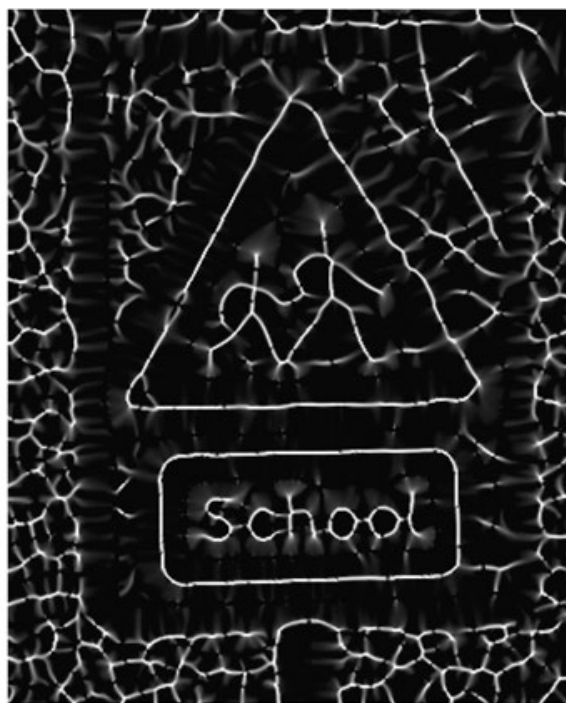

(b)

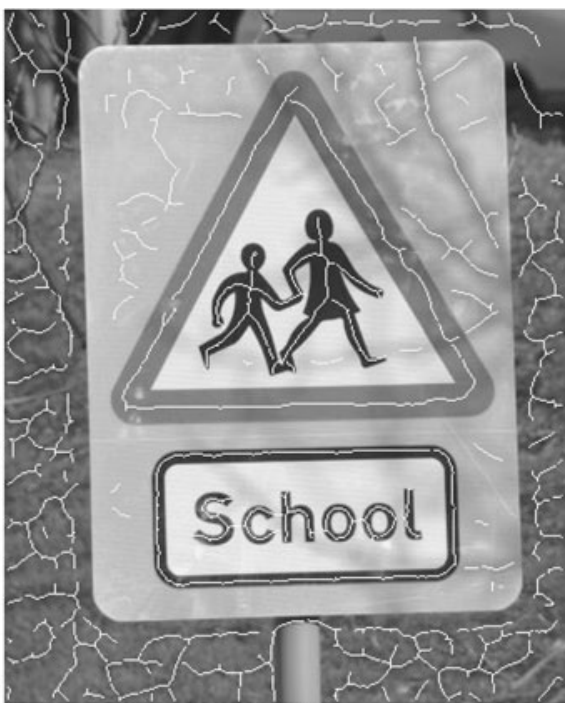

(c)

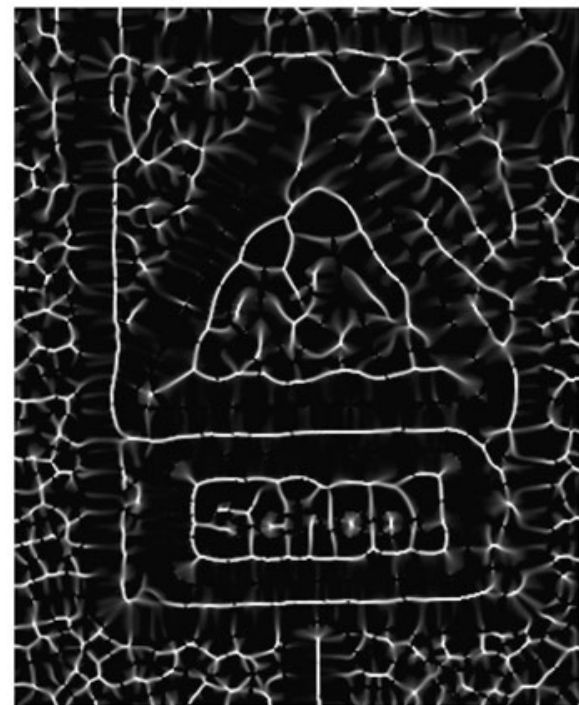

(d)

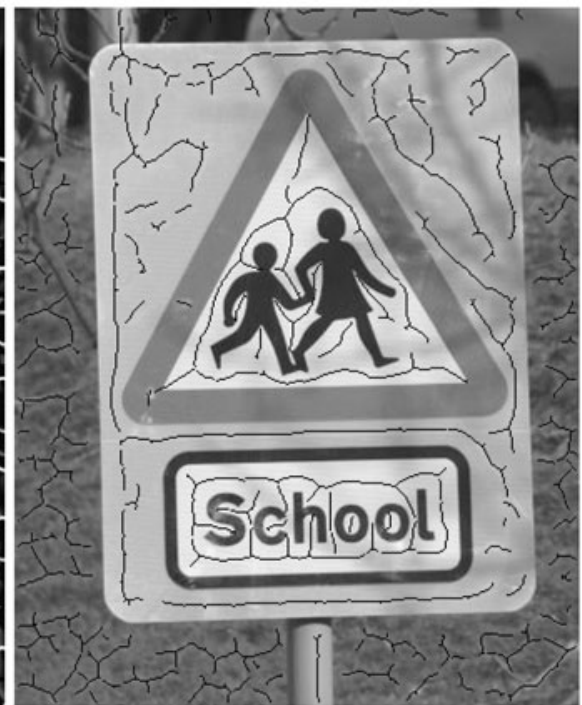

(e)
Fig. 21 Skeleton extraction in a gray-scale traffic sign image of size $431 \times 348$. (a) The traffic sign represents the presence of a school, (b) skeleton strength map for darker regions $(S S M>0)$, (c) binary

automatically selecting the diffusion time and the conduction coefficient. We will extend our approach to 3D volumes for curve skeleton extraction.

Acknowledgements This work is supported by an Innovation Partnership between Sony-Toshiba-IBM and Enterprise Ireland (IP2007-505) and forms part of Trinity's Sony-Toshiba-IBM European Cell/B.E. Center of Competence.

\section{References}

Arcelli, C., \& Baja, G. S. (1992). Ridge points in Euclidean distance maps. Pattern Recognition Letters, 13, 237-243. skeleton for $S S M>0$, (d) skeleton strength map for brighter regions $(S S M<0),(\mathbf{e})$ binary skeleton for $S S M<0$

Aslan, C., Erdem, A., Erdem, E., \& Tari, S. (2008). Disconnected skeleton: shape at its absolute scale. IEEE Transactions on Pattern Analysis and Machine Intelligence, 30(12), 2188-2203.

Bai, X., Latecki, L. J., \& Liu, W. Y. (2007). Skeleton pruning by contour partitioning with discrete curve evolution. IEEE Transactions on Pattern Analysis and Machine Intelligence, 29(3), 449-462.

Blum, H. (1967). A transformation for extracting new descriptors of shape. Models for the Perception of Speech and Visual Form, 363380.

Direkoglu, C. (2009). Feature extraction via heat flow analogy. PhD thesis, University of Southampton, UK.

Direkoglu, C., \& Nixon, M. S. (2007). Shape extraction via heat flow analogy. In Proc. int'l. conf. advanced concepts for intelligent vision systems (Vol. 4678, pp. 553-564).

Direkoglu, C., Dahyot, R., \& Manzke, M. (2010). Skeleton extraction via anisotropic heat flow. In Proceedings of the British 
machine vision conference (pp. 61.1-61.11). Guildford: BMVA Press. ISBN 1-901725-40-5. doi:10.5244/C.24.61.

Gorelick, L., Galun, M., \& Brandt, A. (2006). Shape representation and classification using the Poisson equation. IEEE Transactions on Pattern Analysis and Machine Intelligence, 28(12), 1991-2005.

Grogorishin, T., Abdel-Hamid, G., \& Yang, Y. H. (1996). Skeletonization: an electrostatic field-based approach. Pattern Analysis \& Applications, 1(3), 163-177.

Hassouna, M. S., \& Farag, A. A. (2005). Robust centerline extraction framework using level sets. In Proc. IEEE int'l. conf. computer vision and pattern recognition (pp. 458-465).

Hummel, R. (1986). Representations based on zero-crossings in scalespace. In Proc. IEEE int'l. conf. computer vision and pattern recognition (pp. 204-209).

Kimia, B. B., \& Siddiqi, K. (1994). Geometric heat equation and nonlinear diffusion of shapes and images. In Proc. IEEE int'l. computer vision and pattern recognition (pp. 113-120).

Kimmel, R., Shaked, D., Kiryati, N., \& Bruckstein, A. M. (1995). Skeletonization via distance maps and level sets. Computer Vision and Image Understanding, 62(3), 382-391.

Koenderink, J. (1984). The structure of images. Biological Cybernetics, 50, 363-370.

Krinidis, S., \& Chatzis, V. (2009). A skeleton family generator via physics-based deformable models. IEEE Transactions on Image Processing, 18(1), 1-11.

Lam, L., Lee, S. W., \& Suen, C. Y. (1992). Thinning methodologiesa comprehensive survey. IEEE Transactions on Pattern Analysis and Machine Intelligence, 14(9), 869-885.

Latecki, L. J., Lakamper, R., \& Eckhardt, T. (2000). Shape descriptors for non-rigid shapes with a single closed contour. In Proc. IEEE int'l. conf. computer vision and pattern recognition (pp. 424 429).

Le Bourgeois, F., \& Emptoz, H. (2007). Skeletonization by gradient diffusion and regularization. In Proc. IEEE int'l. conf. image processing (Vol. 3, pp. 33-36).

Lindeberg, T. (1998). Edge detection and ridge detection with automatic scale selection. International Journal of Computer Vision, $30(2), 117-154$.
Macrini, D., Siddiqi, K., \& Dickinson, S. (2008). From skeletons to bone graphs: medial abstraction for object recognition. In Proc. IEEE int'l. conf. computer vision and pattern recognition (pp. 1$8)$.

Malandain, G., \& Vidal, S. F. (1998). Euclidean skeletons. Image and Vision Computing, 16(5), 317-327.

Manay, S., \& Yezzi, A. (2003). Anti-geometric diffusion for adaptive thresholding and fast segmentation. IEEE Transactions on Image Processing, 12(11), 1310-1323.

Ogniewicz, R. L., \& Kubler, O. (1995). Hierarchic Voronoi skeletons. Pattern Recognition, 28(3), 343-359.

Shen, W., Bai, X., Hu, R., Wang, H., \& Latecki, L. J. (2011). Skeleton growing and pruning with bending potential ratio. Pattern Recognition, 44(2), 196-209.

Siddiqi, K., Bouix, S., Tannenbaum, A., \& Zucker, S. W. (1999). The Hamilton-Jacobi skeleton. In Proc. int'l. conf. computer vision (pp. 828-834).

Tari, S., Shah, J., \& Pien, H. (1997). Extraction of shape skeletons from gray-scale images. Computer Vision and Image Understanding, 66(2), 133-146.

Ursell, T. (2007). The diffusion equation-a multi-dimensional tutorial. http://www.rpgroup.caltech.edu/ natsirt/aph162/diffusion. pdf.

Ward, A. D., \& Hamarneh, G. (2010). The groupwise medial axis transform for fuzzy skeletonization and pruning. IEEE Transactions on Pattern Analysis and Machine Intelligence, 32(6), 1084-1096.

Witkin, A. (1983). Scale-space filtering. In Proc. int'l. joint conf. artificial intelligence (pp. 1019-1021).

Xu, C., \& Prince, J. L. (1998). Snakes, shapes and gradient vector flow. IEEE Transactions on Image Processing, 7(3), 359-369.

Yu, Z., \& Bajaj, C. (2004). A segmentation-free approach for skeletonization of gray-scale images via anisotropic vector diffusion. In Proc. IEEE int'l. conf. computer vision and pattern recognition (pp. 415-420). 